\title{
A๖】105 9ь89ट8
}

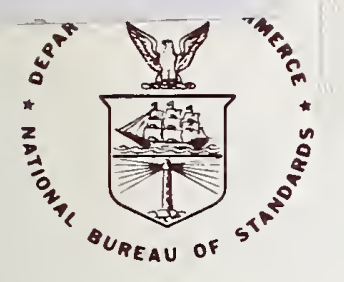

NBS

Publi -

cations

\section{NBS TECHNICAL NOTE 1068-2}

U.S. DEPARTMENT OF COMMERCE / National Bureau of Standards

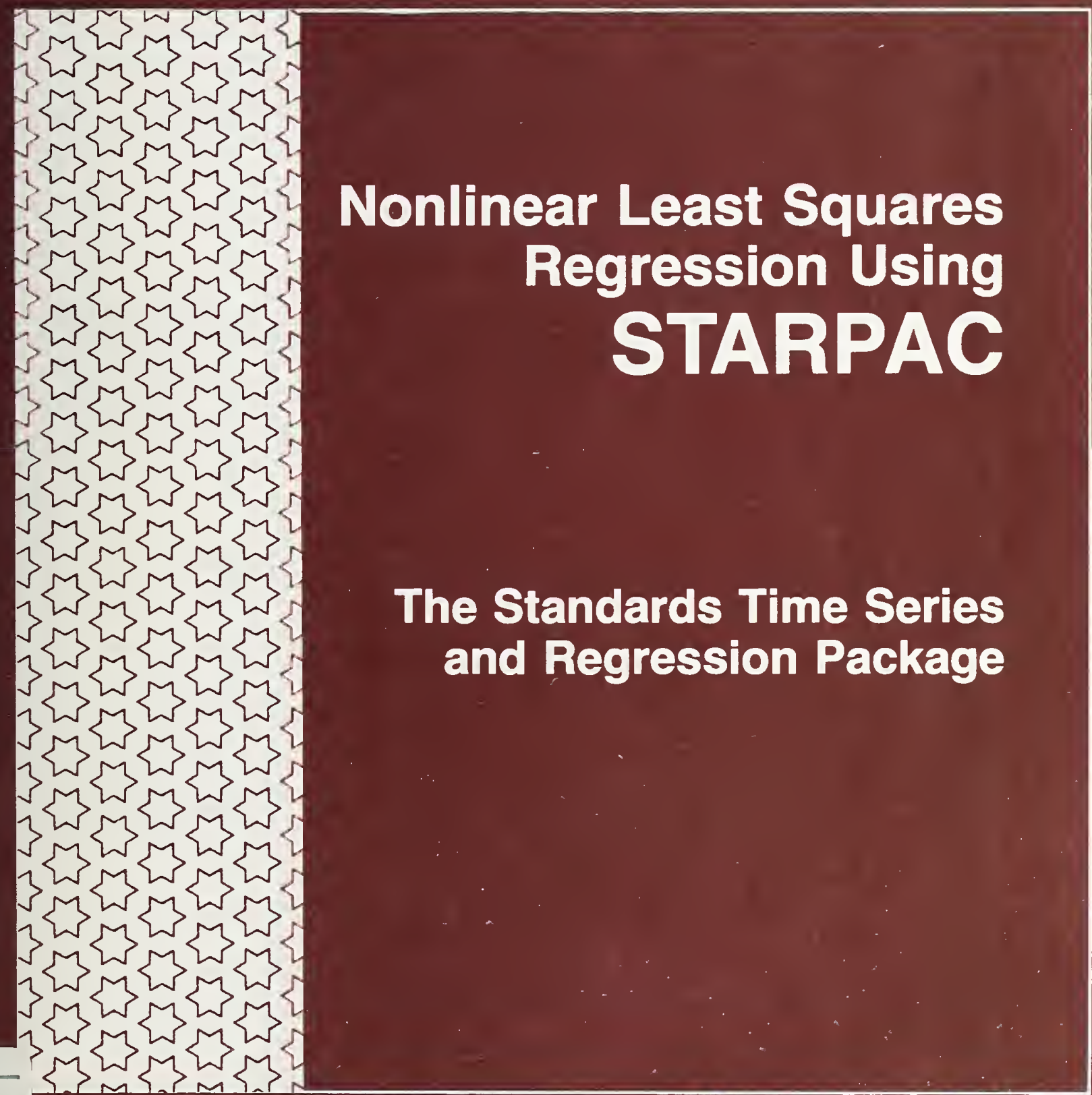




\section{NATIONAL BUREAU OF STANDARDS}

The National Bureau of Standards' was established by an act of Congress on March 3, 190I. The Bureau's overall goal is to strengthen and advance the Nation's science and technology and facilitate their effective application for public benetit. To this end, the Bureau conducts research and provides: (I) a basis for the Nation's physical measurement system, (2) scientific and technological services for industry and government, (3) a technical basis for equity in trade, and (4) technical services to promote public safety. The Bureau's technical work is performed by the National Measurement Laboratory, the National Engineering Laboratory, and the Institute for Computer Sciences and Technology.

THE NATIONAL MEASUREMENT LABORATORY provides the national system of physical and chemical and materials measurement; coordinates the system with measurement systems of other nations and furnishes essential services leading to accurate and uniform physical and chemical measurement throughout the Nation's scientific community, industry, and commerce; conducts materials research leading to improved methods of measurement, standards, and data on the properties of materials needed by industry, commerce, educational institutions, and Government; provides advisory and research services to other Government agencies; develops, produces, and distributes Standard Reference Materials; and provides calibration services. The Laboratory consists of the following centers:

\section{Absolute Physical Quantities ${ }^{2}$ - Radiation Research - Chemical Physics - Analytical Chemistry - Materials Science}

THE NATIONAL ENGINEERING LABORATORY provides technology and technical services to the public and private sectors to address national needs and to solve national problems; conducts research in engineering and applied science in support of these efforts; builds and maintains competence in the necessary disciplines required to carry out this research and technical service; develops engineering data and measurement capabilities; provides engineering measurement traceability services; develops test methods and proposes engineering standards and code changes; develops and proposes new engineering practices; and develops and improves mechanisms to transfer results of its research to the ultimate user. The Laboratory consists of the following centers:

Applied Mathematics - Electronics and Electrical Engineering ${ }^{2}$ - Manufacturing Engineering - Building Technology - Fire Research - Chemical Engineering ${ }^{2}$

THE INSTITUTE FOR COMPUTER SCIENCES AND TECHNOLOGY conducts research and provides scientific and technical services to aid Federal agencies in the selection, acquisition, application, and use of computer technology to improve effectiveness and economy in Government operations in accordance with Public Law 89-306 (40 U.S.C. 759), relevant Executive Orders, and other directives; carries out this mission by managing the Federal Information Processing Standards Program, developing Federal ADP standards guidelines, and managing Federal participation in ADP voluntary standardization activities; provides scientific and technological advisory services and assistance to Federal agencies; and provides the technical foundation for computer-related policies of the Federal Government. The Institute consists of the following centers:

Programming Science and Technology - Computer Systems Engineering.

'Headquarters and Laboratories at Gaithersburg, MD, unless otherwise noted; mailing address Washington, DC 20234.

${ }^{2}$ Some divisions within the center are located at Boulder, CO 80303. 


\title{
Nonlinear Least Squares Regression Using STARPAC
}

\section{The Standards Times Series and Regression Package}

\author{
Janet R. Donaldson
}

Peter V. Tryon

Statistical Engineering Division

Center for Applied Mathematics

National Engineering Laboratory

National Bureau of Standards

Boulder, Colorado 80303

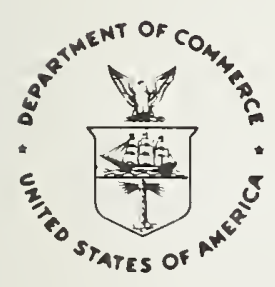

U.S. DEPARTMENT OF COMMERCE, Malcolm Baldrige, Secretary

NATIONAL BUREAU OF STANDARDS, Ernest Ambler, Director 
National Bureau of Standards Technical Note 1068-2

Natl. Bur. Stand. (U.S.), Tech. Note 1068-2, 64 pages (Oct. 1983)

CODEN: NBTNAE

U.S. GOVERNMENT PRINTING OFFICE

WASHINGTON: 1983

For sale by the Superintendent of Documents, U.S. Government Printing Office, Washington, DC 20402

Price $\$ 4.50$

(Add 25 percent for other than U.S. mailing) 
In memory of

Peter V. Tryon

1941 - 1982 


\section{Disclaimer}

No warranties, express or implied, are made by the distributors or developers that STARPAC or its constituent parts are free of error. They should not he relied upon as the sole basis for solving a problem whose incorrect solution could result in injury to person or property. If the programs are employed in such a manner, it is at the user's oun risk and the distrihutors and developers disclaim all liability for such misuse.

Computers have been identified in this paper in order to adequately specify the sample programs and test results. such identification does not imply recommendation or endorsement by the National Bureau of standards, nor does it imply that the equipment identified is necessarily the best available for the purpose. 
STARPAC, the Standards Time Series and Regression Package, is a library of Fortran subroutines for statistica $\overline{1}$ data analysis developed by the Statistical Engineering Division (SED) of the National Bureau of Standards (NBS), Boulder, Colorado. Earlier versions of this library were distributed by the SED under the name STATLIB [Tryon and Donaldson, 1978]. STARPAC incorporates many changes to STATLIB, including additional statistical techniques, improved algorithms and enhanced portability.

We are distributing STARPAC source code in segments, and the documentation as a series of NBS Technical Notes. This will allow us to make the new capabilities of STARPAC avallable more quickly and to facilitate the release of future changes.

The first of the series of Technical Note documenting STARPAC is an Introduction to STARPAC [Donaldson and Tryon, 1983] which gives an an overview of the STARPAC library, defines conventions used to specify the documentation, and presents general background material. The Introduction to STARPAC incudes information which is essential for using the STARPAC 1ibrary, and users should be familiar with its contents before attempting to use any STARPAC subroutine.

This Technical Note documents the STARPAC nonlinear least squares regression subroutines. These subroutines:

- use the quasi-Newton NL2SOL algorithm [Dennis et a1., 1981a], designed specifically for problems which may have highly nonlinear models, large residuals, or poor scaling;

- accept user-supplied derivatives (the Jacobian matrix) when available, and approximate the derivatives numerically otherwise;

- provide utility procedures to verify the correctness of the user-supplied derivatives, or to select optimum step sizes for numerically approximating the derivative [Schnabel, 1982];

- allow both weighted and unweighted analyses;

- permit subsets of the model parameters to be treated as constants with their values held fixed at their input values, allowing the user to examine the results obtained by estimating subsets of the parameters of a general model without rewriting the model subroutine;

- are easy to use, providing three levels of user-control of the computations and results, extensive error handling facilities, comprehensive printed reports, and no size restrictions other than effective machine size; 
- are portable; and

- are easily used with other Fortran subroutine libraries.

Other code segments of STARPAC include subroutines for time series analysis (in both time and frequency domains), line printer graphics, basic statistical analysis, linear least squares regression, and nonlinear optimization. Users may obtain a list of the available code segments and their associated documentation from their local installer of STARPAC, or directly from the author. Notation, format, and naming conventions will remain constant throughout the series of Technical Notes documenting STARPAC, allowing the documentation for each code segment to be used alone or in conjunction with the documentation for another.

The code for STARPAC was developed at the U.S. Department of Commerce Boulder Laboratories on a CDC CYBER 170/750 under NOS 1.4, and all examples presented in this documentation were executed in this environment using the FTN 4.8+552 compiler with rounding. STARPAC has also been tested on the following equipment.

Computer

IBM 4340

VAX $11 / 780$

Sperry $1100 / 82$

Perkin-E1mer 3230

Data General ECLIPSE S/140

\section{$\underline{\text { Facility }}$}

National Center for Atmospheric Research, Boulder, Colorado National Bureau of Standards,
Gaithersburg, Maryland

National Bureau of Standards, Boulder, Colorado

Forest Fire Laboratory, Riverside, California

STARPAC is written in Fortran 166. Adherence to a portable subset of ANSI Fortran [1966] has been verified by the PFORT Verifier [Ryder, 1974] . Workspace and machine-dependent constants are supplied using subroutines based on the Bell Laboratories "Framework for a Portable Library" [Fox et a1., 1978a]. We have also used subroutines from LINPACK [Dongarra et al., 1979], from the "Basic Linear Algebra Subprograms for Fortran Usage" [Lawson et a1., 1979], and from DATAPAC [Fi11iben, 1977]. The subroutines used to compute the least squares solution are those referenced in Dennis et al. [1981a and $1981 \mathrm{~b}]$, and the algorithms used to select optimum step sizes for numerical derivatives, and to check analytic derivatives were developed by Schnabel [1982]. The printed reports from the nonlinear least squares subroutines have been adapted from OMNITAB II [Hogben et a1., 1971]. 
Computer facilities for the STARPAC project have been provided in part by the NOAA Computer Services Division, Boulder, Colorado, and we gratefully acknowledge their support. The STARPAC subroutine library is the result of the programming efforts of Janet $R$. Donaldson and John E. Koontz, with assistance from Ginger A. Caldwell, Steven M. Reefer, and Linda L. Mitchell. Valuable contributions have also been made by each of the members of the Statistical Engineering Division in Boulder, and from many within the STARPAC user community. We are grateful for the many valuable comments that we have received on early drafts of the STARPAC documentation; we wish especially to thank Paul T. Bags, Ginger A. Caldwe11, Sa lIly E. Howe, John E. Koontz, James T. Ringland, Robert B. Schnabel, Ralph M. Slutz, and Dominic F. Vecchia. Finally, we wish to thank Lorna Buhse for excellent manuscript support.

Janet R. Donaldson

Peter V. Tryon (deceased)

Se ptember 1983

vii 
Preface...................................... v

1. Nonlinear Least Squares Regression..................... 2-1.1

A. Introduction. ............................... $2-1.1$

B Subroutine Descriptions........................ 2-1.2

B.1 Nonlinear Least Squares Estimation Subroutines...... 2-1.2

B.2 Derivative Step Size Selection Subroutines......... 2-1.3

B.3 Derivative Checking Subroutines................ 2-1.4

C. Subroutine Declaration and CALL Statements............ 2-1.5

D. Dictionary of Subroutine Arguments and

COMMON Variables............................. 2-1.10

E. Computational Methods.......................... 2-1.20

E.1 Nonlinear Least Squares Estimation.............. 2-1.20

E.1.a The Nonlinear Least Squares Algorithm....... 2-1.20

E.1.b Computed Results and Printed Output From the Nonlinear Least Squares Regression Subroutines....................... 2-1.22

E.2 Step Size Selection....................... 2-1.25

E.2.a The Step Size Selection Algorithm......... 2-1.25

E.2.b Computed Results and Printed Output From the Derivative Step Size Selection Subroutines....................... 2-1.28

E.3 Derivative Checking ..................... 2-1.28

E.3.a The Derivative Checking Algorithm......... 2-1.28

E.3.b Computed Results and Printed Output From the Derivative Checking Subroutines........ 2-1.29

F. Examples................................ 2-1.30

Appendix A...................................... $2-A .1$

Appendix B..................................... $2-B .1$

References..................................... $2-R .1$ 
Nonlinear Least Squares Regression

Using STARPAC

The Standards Time Series and Regression Package

Janet R. Donaldson and Peter V. Tryon

National Bureau of Standards

Washington, DC 20234

STARPAC, the Standards Time Series and Regression Package, is a library of Fortran subroutines for statistical data analysis developed by the Statistical Engineering Division (SED) of the National Bureau of Standards (NBS), Boulder, Colorado. Earlier versions of this library were distributed by the SED under the name STATLIB [Tryon and Donaldson, 1978]. STARPAC incorporates many changes to STATLIB, including additional statistical techniques, improved algorithms and enhanced portability.

STARPAC emphasizes the statistical interpretation of results, and, for this reason, comprehensive printed reports of auxiliary statistical information, often in graphical form, are automatically provided to augment the basic statistical computations performed by each user-callable STARPAC subroutine. STARPAC thus provides the best features of many stand-alone statistical software programs within the flexible environment of a subroutine library.

This Note documents 16 subroutines for nonlinear least squares regression. Twelve of these compute the least squares estimates, performing either weighted or unweighted analysis with either numerically approximated or user-supplied (analytic) derivatives. The other four are user-callable subroutines for two procedures used within the estimation code: the first selects optimum step sizes for approximating the partial derviatives of the model; and the second checks the validity of a user-supplied derivative subroutine.

Key words: derivative checking; NL2SOL; nonlinear least squares; nonlinear regression; quasi-Newton methods; STARPAC; statistical computing; statistical subroutine library; statistics; derivative step size selection; weighted nonlinear least squares. 



\section{CHAPTER 1}

NONLINEAR LEAST SQUARES REGRESSION

\section{A. Introduction}

STARPAC contains 16 user-callable subroutines for nonlinear least squares regression. Twelve of these are estimation subroutines that compute the least squares solution as described below, performing efther weighted or unweighted regression with either numerically approximated or user-supplied (analytic) derivatives. The estimation subroutines allow three levels of control of the computations and printed output, and allow the user to specify a subset of the parameters to be treated as constants, with their values held fixed at their input values. This last feature allows the user to examine the results obtained estimating various subsets of the parameters of a general model without rewriting the model subroutine for each subset. The other four subroutines described in this chapter are utility procedures which choose optimum step sizes for numerically approximating the derivative, and which verify the correctness of user-supplied (analytic) derivatives.

Each of the subroutines described in this chapter assumes that the observations of the dependent variable, $y_{i}$, are modeled by

$$
y_{i}=f_{i}\left(x_{i}, \beta\right)+\varepsilon_{i} \quad \text { for } i=1, \ldots, N \text {, }
$$

where

$\mathrm{N} \quad$ is the number of observations;

$f_{i} \quad$ is the function (nonlinear in $i t s$ parameters) that models the $i^{\text {th }}$ observation;

$x_{i} \quad i s$ the vector of the $M$ independent variables at the $i^{\text {th }}$ observation;

$\beta \quad$ is the vector of the NPAR model parameters; and

$\varepsilon_{i} \quad$ is the unobservable random error in the $i^{\text {th }}$ observation, which is estimated by the $i^{\text {th }}$ residual.

The least squares estimates of the parameters, $\beta$, are obtained using an iterative procedure that requires the matrix of partial derivatives of the model with respect to each parameter,

$$
D(i, k)=\partial f_{i}\left(x_{1}, \beta\right) / \partial \beta(k) \quad \begin{aligned}
& \text { for } i=1, \ldots, N \\
& \text { and } k=1, \ldots, N \text { NPAR }
\end{aligned}
$$

The derivative matrix may be supplied analytically or approximated nume rically. 
The least squares solution, $\beta$, is that which minimizes (with respect to $B$ ) the residual sum of squares function,

$$
\operatorname{RSS}(\beta)=\sum_{i=1}^{N}\left[\left(y_{i}-f_{i}\left(x_{i}, \beta\right)\right)^{2} \cdot w t_{i}\right]=\sum_{i=1}^{N}\left[\varepsilon_{i}^{2} \cdot w t_{i}\right]
$$

where carat ( $\left.{ }^{\wedge}\right)$ denotes the estimated quantity, and

$w t_{i}$ is the weight assigned to the $i^{t h}$ observation $\left(w t_{i}=1.0\right.$ in the "unweighted" case). Appendix A discusses several common applications for weighted least squares.

The user must supply both initial values for the parameters, and a subroutine [see $\S D$, argument NLSMDL] to compute $f_{i}\left(x_{i}, \beta_{\ell}\right), i=1, \ldots, N$, i.e., the predicted values of the dependent variable given the independent variables and the parameter values from iteration $\ell, \ell=1,2, \ldots$. Initial parameter values should be chosen with care, since good values can significantly reduce computer time.

STARPAC provides a variety of subroutines to accommodate many levels of user sophistication and problem difficulty. Users are directed to §B for a brief description of the features and subroutines available. The actual declaration and CALL statements are given in $\S C$, and the subroutine arguments are defined in §D. Generally, the computational details provided in §E are not needed unless difficulties arise. Sample programs and their output are shown in $\S \mathrm{F}$.

\section{B. Subroutine Descriptions}

\section{B.1 Nonlinear Least Squares Estimation Subroutines}

The simplest of the 12 nonlinear least squares estimation subroutines, NLS, requires neither user-supplied weights nor analytic derivatives. The estimated results and a variety of statistics are automatically summarized in a five-part printed report, and the estimated parameters and residuals are returned to the user via the subroutine argument 1 ist (level one control, described below). Most nonlinear least squares problems can be solved using NLS.

The other 11 estimation subroutines add the weighting, derivative, and level two and three control features both singly and in combination, providing greater flexibility to the user at the price of less simplicity. These features are indicated by the suffix letter(s) on the subroutine name (e.g・, NLSS and NLSWDC). 
- Suffix W indicates user-supplied weights.

- Suffix D indicates user-supplied (analytic) derivatives.

- Suffix C indicates level two control of the computations.

- Suffix S indicates level three control of the computations.

The three levels of computation and printed output control are described as follows.

- In level one, a five-part printed report is automatically provided [see $\S E .1 . b]$, and the estimated model parameters and residuals are returned to the user via the argument 1 ist.

- Level two also returns the estimated parameters and residuals, and, in addition, allows the user to supply arguments to indicate

- a subset of the model parameters to be treated as constants, with their values held fixed at their input values;

- either the step sizes used to compute the numerical approximations to the derivative [see $\S .2$ ], or, when user-supplied analytic derivatives are used, whether they will be checked [see $\$ E .3$ ];

- the maximum number of iterations allowed;

- the convergence criteria;

- the scale (i.e., the typical size) of each parameter;

- the maximum change allowed in the parameters at the first iteration;

- how the variance-covariance matrix is to be approximated; and

- the amount of printed output desired.

- Level three has all the features of level two, and, in addition returns the following estimated values via the argument 1ist:

- the number of nonzero weighted observations (only when a weighted analysis is performed);

- the number of parameters actually estimated;

- the residual standard deviation;

- the predicted values;

- the standard deviations of the predicted values;

- the standardized residuals; and

- the variance-covariance matrix of the estimated parameters.

\section{B.2 Derivative Step Size Selection Subroutines}

When the partial derivatives used in the nonlinear least squares solution are not available analytically, STARPAC subroutines approximate them numerically. In this case, the subroutines can select optimum step sizes for approximating the derivatives [see §E.2]. The user also has the option of computing these step sizes independently of the estimation process by calling either of the two step size selection subroutines directly. For example, when 
planning to use the parameter fixing capability [see §D, argument IFIXED] to examine several subsets of the parameters of a general model, computing the step sizes first and passing them to the estimation subroutine is more efficient than recomputing them each time the estimation subroutine is called.

The simplest of the two user-callable step size selection subroutines, STPLS, summarizes the step size selection information for each parameter in a printed report, and returns the step sizes to the user via the subroutine a rgument list.

The second step size selection subroutine, STPLSC, differs from STPLS only in that it enables the user to supply arguments to indicate

- the number of reliable digits in the model results;

- the number of exemptions allowed by the acceptance criteria;

- the scale (i.e., the typical size) of each parameter; and

- the amount of printed output desired.

\section{B.3 Derivative Checking Subroutines}

When the partial derivatives used in the nonlinear least squares solution are available analytically, the user can code them for use by the estimation subroutines [see $\S D$, argument NLSDRV]. Because coding errors are a common problem with user-supplied derivatives, the STARPAC estimation subroutines a utomatically check the validity of the user-supplied derivative code by comparing its results to numerically approximated values for the derivative. When the results are questionable, the checking procedure attempts to determine whether the problem lies with the user's code or with the accuracy of the numerical approximation [see $§ E .3]$. Although the checking procedure is a tomatically available to the estimation subroutines which accept user-supplied derivatives, the user may want to check the derivative code independently of the estimation process. In these cases, the user can call either of the two derivative checking subroutines directly, and suppress checking by the estimation subroutines. [See $\S \mathrm{D}$, argument IDRVCK.]

The simplest of the two derivative checking subroutines, DCKLS, summarizes the results of the check in a printed report.

The second derivative checking subroutine, DCKLSC, differs from DCKLS only in that it enables the user to supply arguments to indicate

- the number of reliable digits in the model results;

- the agreement tolerance;

- the scale (i.e., the typical size) of each parameter;

- the row at which the derivative is to be checked; and

- the amount of printed output desired. 


\section{Subroutine Declaration and CALL Statements}

NOTE: Argument definitions and sample programs are given in $\S D$ and $\S$, respectively. The conventions used to present the following declaration and CALL statments are given in the Introduction to STARPAC [Donaldson and Tryon, 1983, chapter $1, \S B$, and $\S D]$.

\section{Nonlinear Least Squares Estimation Subroutines}

The 〈basic declaration block〉 identifies declaration statements that are needed by all of the nonlinear least squares estimation subroutines. The user should substitute the following four statements for each occurrence of <basic declaration block> given below.

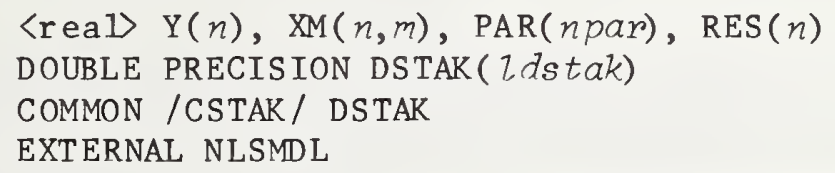

$$
==
$$

NLS: IInweighted nonlinear least squares regression; numerically approximated derivatives; five-part printed report; minimal return of results.

〈basic declaration block〉

$\vdots$

CALL NLS (Y, XM, N, M, IXM, NLSMDL, PAR, NPAR, RES, LDSTAK)

$$
===
$$

NLSC: Inweighted nonlinear least squares regression; numerically approximated derivatives; user-supplied control values; minimal return of results.

\section{〈basic declaration block〉}

INTEGER IFIXED( npar)

〈real〉STP(npar), STOPSS, STOPP, SCALE(npar), DELTA

$\vdots$

CALL NLSC ( $Y, X M, N, M$, IXM, NLSMDL, PAR, NPAR, RES, LDSTAK,

1 IFIXED, STP, MIT, STOPSS, STOPP, SCALE, DELTA, IVAPRX, NPRT)

$$
==
$$


NLSS: IInweighted nonlinear least squares regression; numerically

approximated derivatives; user-supplied control values; maximal return

of results.

〈basic declaration block〉

INTEGER IFIXED( $n$ par)

$\langle$ rea1〉STP(npar), STOPSS, STOPP, SCALE( $n$ par), DELTA

$\langle$ real〉 RSD, $\operatorname{PV}(n), \operatorname{SDPV}(n), \operatorname{SDRES}(n), \operatorname{VCV}($ npare,npare)

;

CALL NLSS ( $Y, X M, N, M$, IXM, NLSMDL, PAR, NPAR, RES, LDSTAK,

1 IFIXED, STP, MIT, STOPSS, STOPP, SCALE, DELTA, IVAPRX, NPRT,

2 NPARE, RSD, PV, SDPV, SDRES, VCV, IVCV)

NLSW: Weighted nonlinear least squares regression; numerically approximated derivatives; five-part printed report; minimal return of results.

〈basic declaration block〉

$\langle$ rea1〉 WT $(n)$

$\vdots$

CALL NLSW (Y, WT, XM, N, M, IXM, NLSMDL, PAR, NPAR, RES, LDSTAK)

$===$

NLSWC: Weighted nonlinear least squares regression; nimerically approximated derivatives; user-supplied control values; minimal return of results.

〈basic declaration block>

INTEGER IFIXED(npar)

$\langle$ rea1〉 $\mathrm{WT}(n)$

〈rea1〉 STP(npar), STOPSS, STOPP, SCALE(npar), DELTA

$\vdots$

CALL NLSWC ( $Y$, WT, XM, N, M, IXM, NLSMDL, PAR, NPAR, RES, LDSTAK, 1 IFIXED, STP, MIT, STOPSS, STOPP, SCALE, DELTA, IVAPRX, NPRT) 
NLSWS: Weighted nonlinear least squares regression; numerically approximated derivatives; user-supplied control values; maximal return of results.

〈basic declaration block>

INTEGER IFIXED(npar)

$\langle$ real〉 WT $(n)$

〈real〉 STP(npar), STOPSS, STOPP, SCALE(npar), DELTA

$\langle$ real〉 RSD, $\operatorname{PV}(n), \operatorname{SDPV}(n), \operatorname{SDRES}(n), \operatorname{VCV}($ npare,npare)

$\vdots$

CALL NLSWS ( $Y$, WT, XM, N, M, IXM, NLSMDL, PAR, NPAR, RES, LDSTAK,

1 IFIXED, STP, MIT, STOPSS, STOPP, SCALE, DELTA, IVAPRX, NPRT,

2 NNZW, NPARE, RSD, PV, SDPV, SDRES, VCV, IVCV)

NLSD: Unweighted nonlinear least squares regression; user-supplied

derivatives; five-part printed report; minimal return of results.

〈basic declaration block〉

EXTERNAL NLSDRV

$\vdots$

CALL NLSD ( $Y, X M, N, M, I X M, N L S M D L, N L S D R V, P A R, N P A R, R E S$, 1 LDSTAK)

$==\boldsymbol{=}$

NLSDC: Inweighted nonlinear least squares regression; user-supplied derivatives; user-supplied control values; minimal return of results.

〈basic declaration block〉

EXTERNAL NLSDRV

INTEGER IFIXED( $n$ par)

〈real〉 STOPSS, STOPP, SCALE(npar), DELTA

$\vdots$

CALL NLSDC ( $Y, X M, N, M$, IXM, NLSMDL, NLSDRV, PAR, NPAR, RES, 1 LDSTAK, IFIXED, IDRVCK, MIT, STOPSS, STOPP, SCALE, DELTA,

2 IVAPRX, NPRT) 
NLSDS: Inweighted nonlinear least squares regression; user-supplied derivatives; user-supplied control values; maximal return of results.

〈basic declaration block>

EXTERNAL NLSDRV

INTEGER IFIXED(npar)

〈rea1〉 STOPSS, STOPP, SCALE(npar), DELTA

$\langle$ rea1〉 RSD, $\operatorname{PV}(n), \operatorname{SDPV}(n), \operatorname{SDRES}(n), \operatorname{VCV}($ npare,npare)

$\vdots$

CALL NLSDS ( $Y, X M, N, M, I X M, N L S M D L, ~ N L S D R V, P A R, N P A R$, RES, 1 LDSTAK, IFIXED, IDRVCK, MIT, STOPSS, STOPP, SCALE, DELTA, 2 IVAPRX, NPRT, NPARE, RSD, PV, SDPV, SDRES, VCV, IVCV)

$$
===
$$

NLSWD: Weighted nonlinear least squares regression; user-supplied derivatives; five-part printed report; minimal return of results.

〈basic declaration block〉

EXTERNAL NLSDRV

$\langle$ real〉 WT $(n)$

$\vdots$

CALL NLSWD (Y, WT, XM, N, M, IXM, NLSMDL, NLSDRV, PAR, NPAR, RES, 1 LDSTAK)

NLSWDC: Weighted nonlinear least squares regression; user-supplied

derivatives; user-supplied control values; minimal return of results.

〈basic declaration block>

EXTERNAL NLSDRV

INTEGER IFIXED( npar)

$\langle$ real〉 WT $(n)$

〈real〉 STOPSS, STOPP, SCALE(npar), DELTA

$\vdots$

CALL NLSWDC (Y, WT, XM, N, M, IXM, NLSMDL, NLSDRV, PAR, NPAR, RES, 1 LDSTAK, IFIXED, IDRVCK, MIT, STOPSS, STOPP, SCALE, DELTA, 2 IVAPRX, NPRT) 
NLSWDS: Weighted nonlinear least squares regression; user-supplied

derivatives; user-supplied control values; maximal return of results.

〈basic declaration block>

EXTERNAL NLSDRV

INTEGER IFIXE D(npar)

$\langle$ real〉 WT $(n)$

$\langle$ rea1〉 STOPSS, STOPP, SCALE(npar), DELTA

〈real〉 RSD, $\operatorname{PV}(n), \operatorname{SDPV}(n), \operatorname{SDRES}(n), \operatorname{VCV}($ npare,npare)

$$
\vdots
$$

CALL NLSWDS (Y, WT, XM, N, M, IXM, NLSMDL, NLSDRV, PAR, NPAR, RES,

1 LDSTAK, IFIXED, IDRVCK, MIT, STOPSS, STOPP, SCALE, DELTA,

2 IVAPRX, NPRT, NNZW, NPARE, RSD, PV, SDPV, SDRES, VCV, IVCV)

$$
===
$$

\section{Step Size Selection Subroutines}

STPLS: Optimum step size selection for numerically approximating

derivatives; printed report; maximal return of results.

$\langle$ reaI〉 $\operatorname{XM}(n, m), \operatorname{PAR}(n$ par $), \operatorname{STP}(n p a r)$

DOUBLE PRECISION DSTAK( Zdstak)

COMMON /CSTAK/ DSTAK

EXTERNAL NLSMDL

$\vdots$

CALL STPLS (XM, N, M, IXM, NLSMDL, PAR, NPAR, LDSTAK, STP)

$$
===
$$

STPLSC: nptimum step size selection for numerically approximating

derivatives; user-supplied control values; maximal return of results.

$\langle$ rea1〉 XM(n,m), $\operatorname{PAR}(n$ par $), \operatorname{STP}(n$ par $)$

$\langle$ rea1〉 EXMPT, SCALE(npar)

DOUBLE PRECISION DSTAK( $Z$ Astak)

COMMON /CSTAK/ DSTAK

EXTERNAL NLSMDL

$\vdots$

CALL STPLSC (XM, N, M, IXM, NLSMDL, PAR, NPAR, LDSTAK, STP, 1 NETA, EXMPT, SCALE, NPRT) 
DCKLS: Derivative checking; printed report; maximal return of results.

$\langle$ real〉 $\operatorname{XM}(n, m), \operatorname{PAR}(n p a r), \operatorname{STP}(n p a r)$

DOUBLE PRECISION DSTAK ( $て$ dstak)

COMMON /CSTAK/ DSTAK

EXTERNAL NLSMDL, NLSDRV

$\vdots$

CALL DCKLS (XM, N, M, IXM, NLSMDL, NLSDRV, PAR, NPAR, LDSTAK)

DCKLSC: Derivative checking; user-supplied control values; maximal return of results.

$\langle$ real〉 XM(n,m), $\operatorname{PAR}(n p a r), \operatorname{STP}(n p a r)$

$\langle$ real〉 SCALE $(n$ par $)$

DOUBLE PRECISION DSTAK( $(d s t a k)$

COMMON /CSTAK/ DSTAK

EXTERNAL NLSMDL, NLSDRV

$\vdots$

CALL DCKLSC (XM, N, M, IXM, NLSMDL, NLSDRV, PAR, NPAR, LDSTAK, 1 NETA, NTAU, SCALE, NROW, NPRT)

$== \pm$

D. Dictionary of Subroutine Arguments and COMMON Variables

NOTE: $\rightarrow$ indicates that the argument is input to the subroutine, and that the input value is preserved;

$<-$ indicates that the argument is returned by the subroutine;

$\longleftrightarrow->$ indicates that the argument is input to the subroutine and that the input value will be overwritten by the subroutine;

--- indicates that the argument is input to some subroutines and is returned by others;

*** indicates that the argument is a suhroutine name;

-.. indicates that the variable is passed via COMMON.

D <-- The matrix of exact dimension $N$ by NPAR that contains the partial derivatives of the model with respect to each parameter, $\operatorname{PAR}(k), k=1, \ldots$, NPAR. This argument is used within derivative subroutine NLSDRV [see argument NLSDRV below]. 
DELTA $\rightarrow$ The maximum scaled change allowed in the parameters at the first iteration, i.e., $\delta_{0}\left[\right.$ see $\left.\S_{E} .1 . a\right]$. The default value is 100.0 . When DELTA $\leqslant 0.0$, or when DELTA is not an argument of the subroutine CALL statement, the default value is used. A smaller value of DELTA may be appropriate if, at the first iteration, the computation of the predicted values from the user's model subroutine produces an arithmetric overflow, or the parameters leave the region of interest in parameter space. A reasonable alternative to the default value of DELTA is an upper bound to the scaled change that the estimated parameters should be allowed to make on the first iteration,

$\operatorname{DELTA}=\min \left\{\left|\Delta_{\max } \beta(k)\right| / \operatorname{SCALE}(k)\right.$, for $k=1, \ldots$, NPAR $\}$

where $\Delta_{\max } \beta(k)$ is the maximum change allowed for the $k^{\text {th }}$ parameter at the first iteration.

DSTAK $\cdots$ The DOUBLE PRECISION vector in COMMON /CSTAK/ of dimension at least LDSTAK. DSTAK provides workspace for the computations. The first LDSTAK locations of DSTAK will be overwritten during subroutine execution.

EXMPT $\rightarrow$ The proportion used to compute the number of observations, $\alpha=\operatorname{EXMPT} \cdot N$, for which the forward difference quotient derivative with respect to a given parameter is exempted from meeting the acceptance criteria for step size selection [see §E.2.a]. The default value for EXMPT is 0.1 ( 10 percent). When the user-supplied value is outside the range $[0.0,1.0]$, or when EXMPT is not an argument of the subroutine CALL statement, the default value is used.

IDRVCK --> The indicator variable used to designate whether or not the user-supplied derivative subroutine is to be checked. When IDRVCK $\neq 0$ the derivative is checked, and when IDRVCK $=0$ it is not. The default value is IDRVCK $\neq 0$. When IDRVCK is not an argument of the subroutine CALL statement, the default value is used.

IERR - . An error flag returned in COMMON /ERRCHK/. [See Donaldson and Tryon, 1983, chapter 1, §D.5.] Note that using (or not using) the error flag will not affect any error messages that are automatically printed, even when the user has suppressed the norma1 printed output.

For the estimation subroutines:

IERR $=0$ indicates that no errors were detected, and that the iterations converged satisfactorily.

IERR $=1$ indicates that improper input was detected. 
IERR $=2$ indicates that the computation of the residual sum of squares using the initial parameter values produced an arithmetic overflow. The user should reduce the size of DELTA or should supply new starting values.

IERR $=3$ indicates that the model is computationally singular, which means the model has too many parameters near the solution. The user should examine the model and data to determine and remove the cause of the singularity.

IERR $=4$ indicates that at least one of the standardized residuals could not be computed because its standard deviation was zero. The validity of the covariance matrix is questionable.

IERR $=5$ indicates false convergence. [See §E.1.a.]

IERR $=6$ indicates that convergence was not reached in the allowed number of iterations or model subroutine calls. [See argument MIT.]

IERR $=7$ indicates that the variance-covariance matrix could not he computed.

For the step size selection subroutines:

IERR $=0$ indicates that no errors were detected, and that all the step sizes satisfied the selection criteria.

IERR $=1$ indicates that improper input was detected.

IERR $=2$ indicates that one or more of the step sizes did not satisfy the selection criteria.

For the derivative checking subroutines:

IERR $=0$ indicates that no errors were detected, and that the user-supplied derivative code appears to be correct.

IERR $=1$ indicates that improper input was detected.

IERR $=2$ indicates that the user-supplied derivative code and numerical derivatives do not agree for at least one parameter, but that in each case of disagreement the accuracy of the numerical derivatives is questionable. Further testing is suggested.

IERR = 3 indicates that the user-supplied derivative code and numerical derivatives do not agree for at least one parameter, and in at least one instance of disagreement there is no reason to doubt the numerical derivatives.

IFIXED - $\rightarrow$ The vector of dimension at least NPAR that contains values used to indicate whether the corresponding parameter in PAR is to be treated as a fixed constant or is to be estimated. If IFIXED(I) >0, PAR(I) will be held fixed at its input value; if $\operatorname{IFIXED}(I)=0$, PAR(I) will be estimated using the least squares 
procedure described in $\S A$. The default values are $\operatorname{IFIXED}(I)=0$, $I=1, \ldots$, NPAR, i.e., all parameters are estimated. When IFIXED(1) $\leqslant-1$, or when IFIXED is not an argument of the subroutine CALL statement, the default value will be used.

IVCV $\rightarrow$ The exact value of the first dimension of the matrix VCV as specified in the calling program.

IVAPRX $\rightarrow$ The indicator variable used to specify how the variance-covariance matrix, VCV, is to be approximated. Three approximations are available:

(1) $\mathrm{VCV}=\mathrm{RSD}^{2} \cdot(\hat{\mathrm{D}} \cdot \hat{\mathrm{W}} \cdot \hat{\mathrm{D}})$

(2) $\mathrm{VCV}=\mathrm{RSD}^{2} \cdot \mathrm{H}^{-1}$

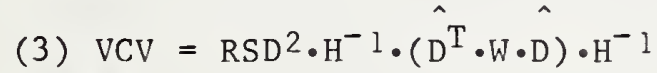

where

$H=\hat{D^{T}} \cdot \hat{W} \cdot \hat{D}+\left\{\sum_{i=1}^{N} \hat{\varepsilon_{i}} \cdot w t_{i} \cdot\left(\partial^{2} \hat{\varepsilon_{i}} / \partial \hat{\beta}(j) \partial \hat{\beta}(k)\right) \begin{array}{l}\text { for } j=1, \ldots, \text { NPAR } \\ \text { and } \hat{k}=1, \ldots, \text { NPAR }\}\end{array}\right.$

$\mathrm{W}$ is an $\mathrm{N}$ by $\mathrm{N}$ diagonal matrix of user-supplied weights,

$W=\operatorname{diag}\left\{w t_{i}, i=1, \ldots, N\right\}$,

when a weighted analysis is performed, and is the identity matrix otherwise.

Approximation (1) is based on the assumption that $\mathrm{H} \sim \hat{\mathrm{D}^{\mathrm{T}}} \cdot \hat{W} \cdot \hat{\mathrm{D}}$ because the residuals are sufficiently small at the solution; approximation (2) is based on the assumption that the necessary conditions for asymptotic maximum likelihood theory have been met; and approximation (3) is based on the assumption that the necessary conditions for asymptotic maximum likelihood theory may be violated. All approximations to the variance-covariance matrix are subject to sampling variation, however, because they are computed using the estimated parameter values. The variance-covariance matrix computed for any particular nonlinear least squares solution should thus be regarded as only a rough estimate, correct to within an order of magnitude [Bard, 1974].

If IVAPRX $=1$ or 4 then approximation ( 1 ) is used;

$=2$ or 5 then approximation (2) is used; and

$=3$ or 6 then approximation (3) is used.

If $\operatorname{IVAPRX}=1,2$, or 3 , then, when user-supplied analytic derivatives are available [see argument NLSDRV], they are used to compute VCV; if IVAPRX $=4,5$, or 6 , then only the predicted values from the model subroutine are used to compute VCV. When analytic derivatives are available, options 1, 2, or 3, will generally result in a faster, more accurate computation of VCV. 
The default value for IVAPRX is 1 . When argument IVAPRX is outside the range $[1,6]$, or when IVAPRX is not an argument of the subroutine CALL statement, then the default value will be used.

IXM - $>$ The exact value of the first dimension of the matrix XM as specified in the calling program.

LDSTAK - $\rightarrow$ The length of the DOUBLE PRECISION workspace vector DSTAK. LDSTAK must equal or exceed the appropriate value given below. If the single precision version of STARPAC is being used, $P=0.5$ otherwise $P=1.0$ [see Donaldson and Tryon, 1983, chapter $1, \S B$ ].

For NLS, NLSC, NLSS, NLSW, NLSWC, NLSWS :

$$
\begin{aligned}
\mathrm{LDSTAK} \geqslant & 27+\max \{\mathrm{IS} \cdot(\mathrm{N}+\mathrm{NPAR}), 30+\mathrm{NPARE}\}+ \\
& \mathrm{P} \cdot \max \left\{\mathrm{IS} \cdot 10 \cdot \mathrm{N}, 94+\mathrm{N} \cdot(3+\mathrm{NPAR})+\left(3 \cdot \mathrm{NPARE}^{2}+37 \cdot \mathrm{NPARE}\right) / 2\right\}
\end{aligned}
$$

where IS $=1$ if default values are used for the derivative step sizes, and $\mathrm{IS}=0$ otherwise.

For NLSD, NLSDC, NLSDS, NLSWD, NLSWDC, NLSWDS :

$$
\mathrm{LDSTAK} \geqslant 45+\mathrm{NPAR}+\mathrm{P} \cdot\left(94+\mathrm{N} \cdot(3+\mathrm{NPAR})+\left(3 \cdot \mathrm{NPARE}^{2}+35 \cdot \mathrm{NPARE}\right) / 2\right)
$$

For STPLS, STPLSC:

$$
\text { LDSTAK } \geqslant 27+(N+N P A R)+P \cdot 10 \cdot N
$$

For DCKLS, DCKLSC:

$$
\mathrm{LDSTAK} \geqslant 14+\mathrm{NPAR}+\mathrm{P} \cdot(\mathrm{N} \cdot \mathrm{NPAR}+\mathrm{N}+\mathrm{NPAR})
$$

M - $\rightarrow$ The number of independent variables, i.e., the number of columns of data in XM.

MIT - $\rightarrow$ The maximum number of iterations allowed. This argument is also used to compute the maximum number of model subroutine calls, $(2 \cdot$ MIT). The iterations will stop if either limit is reached, although, as a rule, the maximum number of iterations will be reached first. The default value for the maximum number of iterations is 21 . When MIT $\leqslant 0$, or when MIT is not an argument of the subroutine CALL statement, the default value will be used.

N $\quad-\rightarrow$ The number of observations. 
$\rightarrow$ The number of reliable decimal digits in the predicted values (PV) computed by the user's model subroutine. The default value for NETA is experimentally determined by the procedure described in Appendix B. The default value will be used when NETA is not an argument in the subroutine CALL statement, or when the user-supplied value of NETA is outside the range [1, DIGITS], where DIGITS is the number of decimal digits carried by the user's computer for a single precision value when the single precision version of STARPAC is being used and is the number carried for a double precision value otherwise.

NLSDRV $* * *$ The name of the user-supplied subroutine that computes the partial derivative matrix (Jacobian). This argument must be listed in an EXTERNAL statement in the program which calls the STARPAC estimation or derivative checking subroutine. The form of the derivative subroutine argument 1 ist and dimensioning statements must be exactly as shown below, although if there is only one independent variable $(M=1)$, XM may be declared to be a vector with dimension IXM.

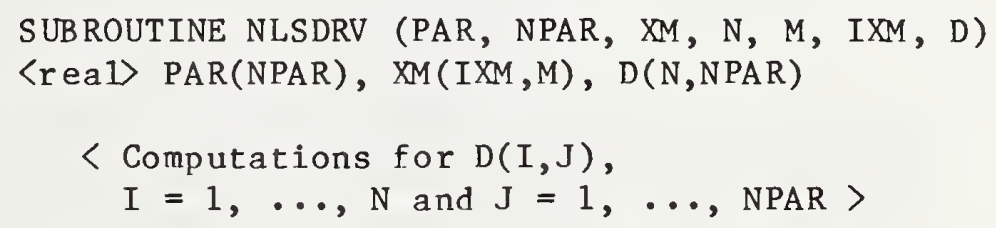

RETURN

END

NLSMDL $* * *$ The name of the user-supplied subroutine that computes the predicted value of the dependent variable given the independent variables and the current values of the model parameters. This argument must be 1 isted in an EXTERNAL statement in the program which calls the STARPAC estimation, step size selection, and/or derivative checking subroutines. The form of the model subroutine argument list and dimensioning statements must be exactly as shown below, although if there is only one independent variable $(M=1)$, XM may be declared to be a vector with dimension IXM.

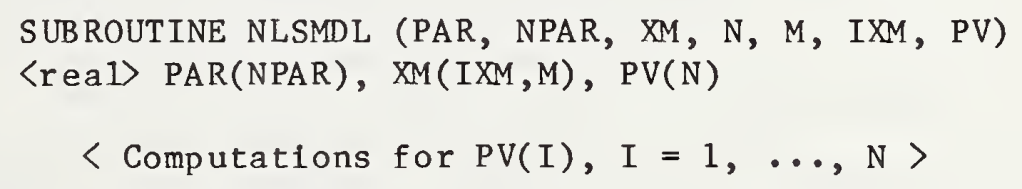

NNZW <- The number of observations with nonzero weights. 
NPAR $\rightarrow$ The number of parameters in the model, including both those held fixed at their starting values, and those which are to be estimated.

NPARE <-- The number of parameters actually estimated, i.e., the number of zero elements in IFIXED. N.B. This value is returned by the estimation subroutines.

NPRT $\quad-\rightarrow$ The argument controling printed output.

For the estimation subroutines:

NPRT is a five-digit integer, in which the value of the $I^{\text {th }}$ digit (counting from left to right) is used to control the $I^{\text {th }}$ section of the output.

If the $I^{\text {th }}$ digit $=0$ the output from the $I^{\text {th }}$ section is suppressed;

$=1$ the brief form of the $\mathrm{I}^{\text {th }}$ section is given;

$\geqslant 2$ the full form of the $\mathrm{I}^{\mathrm{th}}$ section is given.

The default value for NPRT is 11112. When NPRT $\leqslant-1$, or when NPRT is not an argument in the subroutine CALL statement, the default value will he used. If the convergence criteria are not satisfied, the subroutine gives a suitable warning and provides a printed report even if NPRT $=0$. A full discussion of the printed output is given in $\$ E .1 . b$, and is sumarized as follows.

Section 1 lists the starting estimates and control values. Brief output and full output are the same for this section.

Section 2 reports the results of the iterations. Brief output includes information only about the first and last iteration while full output includes information about a11 of the iterations.

Section 3 provides information for each observation based on the final solution. Brief output includes information for the first 40 observations, while full output provides the information for all of the data.

Section 4 is a set of four residual plots. Brief output and full output are the same for this section.

Section 5 is the final summary of the estimated parameters. Brief output does not include printing the variance-covariance matrix, while full output does.

For the step size selection and derivative checking subroutines:

If NPRT $=0$ the output is suppressed.

If NPRT $\neq 0$ the output is provided.

If the acceptance criteria were not met, then a printed report

is provided even if $N P R T=0$. 
$-\rightarrow$ The row of the independent variable matrix at which the user-supplied derivative code is to be checked. The default value is the first row with no independent variables equal to zero; when al1 rows have one or more independent variables equal to zero, row one will be used for the default value. When the user-supplied value is outside the range $[1, N]$, or when NROW is not an argument of the subroutine CALL statement, the default value will be used.

NTAU $\rightarrow$ The agreement tolerance, i.e., the number of digits of agreement required between the user-supplied derivatives and the derivatives numerically approximated by the derivative checking subroutine. The default value is NETA/4. When the user-supplied value of NTAU is outside the range [1, NETA/2], or when NTAU is not an argument of the subroutine CALL statement, the default value will be wised.

PAR --- The vector of dimension at least NPAR that contains the parameter values. For all estimation subroutines it must contain initial values for the parameters on input and will contain the final values on return. For the step size and derivative checking subroutines it must contain the parameter values at which the operations are to be performed.

PV <- The vector of dimension at least $\mathrm{N}$ that contains the predicted values of the dependent variable at the solution,

$P V(i)=f_{i}\left(x_{i}, \beta\right)=y_{i}, \quad$ for $i=1, \ldots, N$.

RES <- The vector of dimension at least $N$ that contains the residuals at the solution,

$\operatorname{RES}(i)=y(i)-f_{i}\left(x_{i}, \beta\right)=y(i)-y(i)=\varepsilon(i)$, for $i=1, \ldots, N$.

RSD <-- The residual standard deviation at the solution, $\operatorname{RSD}=(\operatorname{RSS}(\hat{\beta}) /(\mathrm{NNZW}-\mathrm{NPARE}))^{1 / 2}$.

SCALE $\rightarrow$ The vector of dimension at least NPAR that contains the scale, or typical size, of each parameter. The vector SCALE is used to normalize the size of each parameter so that

$|\beta(j) / \operatorname{SCALE}(j)| \approx|\beta(k) / \operatorname{SCALE}(k)| \quad$ for $k=1, \ldots, N \operatorname{NPAR}$ and $j=1, \ldots$, NPAR.

Values of $|\operatorname{SCALE}(k)|>|\beta(k)|$ can be used to increase the step size in cases where the model function is known to be insensitive to small changes in the value $\beta(k)$.

For the estimation subroutines:

The default values for SCALE are selected by the NL2SOL algorithm [Dennis et al., 1981a and 1981b] and are updated at each iteration. When SCALE is not an argument in the 
subroutine CALL statement, or when the user-supplied value for $\operatorname{SCALE}(1) \leqslant 0$, the default procedure will be used to select scale values. When $\operatorname{SCALE}(1)>0$, values of $\operatorname{SCALE}(k) \leqslant 0$, $k=2, \ldots$. NPAR, will be interpreted as an input error. User-supplied scale values may be either a vector of the typical size of each parameter or a vector of ones if the typical sizes of the parameters are roughly equal; user-supplied scale values can sometimes result in reduced computing time since these values are not updated at each iteration.

For the derivative checking and step size selection subroutines:

The default values of SCALE are defined as:

$\left.\begin{array}{l}\operatorname{SCALE}(k)=1.0 \quad \text { if } \beta(k)=0.0 \\ \operatorname{SCALE}(k)=|\beta(k)| \text { otherwise }\end{array}\right\} \quad$ for $k=1, \ldots, \operatorname{NPAR}$.

When SCALE is not an argument in the subroutine CALL statement, or when the user-supplied value of $|\operatorname{SCALE}(k)| \leqslant|\beta(k)|$, the default value for $\operatorname{SCALE}(k)$ is used. When $\operatorname{SCALE}(1) \leqslant 0$, the default values will be used for $\operatorname{SCALE}(k), k=1, \ldots$, NPAR. When $\operatorname{SCALE}(1)>0$, values of $\operatorname{SCALE}(k) \leqslant 0, k=2, \ldots$, NPAR, will be interpreted as an input error.

SDPV <-- The vector of dimension at least $\mathrm{N}$ that contains an approximation to the standard deviation of each predicted value at the solution,

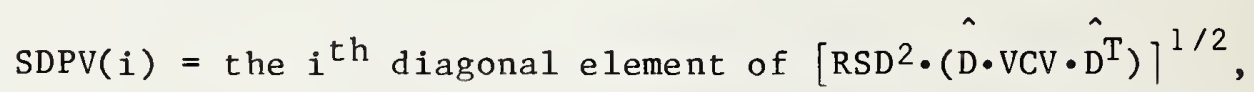

for $i=1, \ldots, N$. This approximation is based on a linearization of the model in the neighborhood of the solution; the validity of the approximation depends on the nonlinearity of the model. This approximation may be extremely inaccurate for a problem with a highly nonlinear model.

SDRES <-- The vector of dimension at least $\mathrm{N}$ that contains an approximation to the standardized residuals at the solution,

$\operatorname{SDRES}(i)=\operatorname{RES}(i) /\left[\left(\operatorname{RSD}^{2} / \mathrm{WT}(i)\right)-\operatorname{SDPV}(i)^{2}\right]^{1 / 2}$,

for $i=1, \ldots, N$, which is the $i^{\text {th }}$ residual divided by its individual estimated standard deviation. This approximation is based on a linearization of the model in the neighborhood of the solution; the validity of the approximation depends on the nonlinearity of the model. This approximation may be extremely inaccurate for a problem with a highly nonlinear model. 
STOPP - $\rightarrow$ The stopping value for the convergence test based on the maximum scaled relative change in the parameters at the most recent iteration. The convergence criterion is satisfied if the current step is a Newton step [see Dennis et a1., 1981a] and

$\frac{\max \left\{\left|\beta_{\ell+1}(k)-\beta_{\ell}(k)\right| / \operatorname{SCALE}(k) \text { for } k=1, \ldots, \text { NPAR }\right\}}{\max \left\{\left(\left|\beta_{\ell+1}(k)\right|+\left|\beta_{\ell}(k)\right|\right) / \operatorname{SCALE}(k) \text { for } k=1, \ldots, \text { NPAR }\right\}}<$ STOPP

This convergence test is roughly equivalent to the test based on the maximum relative change in each parameter as measured by $\max \left\{\left|\beta_{\ell+1}(k)-\beta_{\ell}(k)\right| /\left|\beta_{\ell}(k)\right|\right.$ for $k=1, \ldots$, NPAR $\}$ STOPP is not a scale-dependent value; if its value is $10^{-4}$, then this criteria will be met when the first four digits of each parameter are the same at two successive iterations, regardless of the size of the parameter values.

The default value is approximately $10^{-D I G I T S} / 2$, where DIGITS is the number of decimal digits carried by the user's computer for a single precision value when the single precision version of STARPAC is being used, and is the number carried for a double precision value otherwise. When the user-supplied value for STOPP is outside the interval $[0.0,1.0]$, or when STOPP is not an argument of the subroutine CALL statement, the default value will be used.

STOPSS $\rightarrow$ The stopping value for the convergence test based on the ratio of the forecasted change in the residual sum of squares, $\Delta s^{2} \ell+1$, to the current residual sum of squares, $s_{\ell}^{2}=\operatorname{RSS}\left(\beta_{\ell}\right) /($ NNZW-NPARE). The convergence criterion is satisfied if certain conditions are met [see Dennis et a1., 1981a] and

$\Delta \mathrm{s}_{\ell+1}^{2} / \mathrm{s}^{2} \ell<$ STOPSS.

This convergence test is roughly equivalent to the test based on the relative change in the residual standard deviation between two iterations $\ell$ and $\ell+1$ as measured by $\left(s_{\ell}-s_{\ell+1}\right) / s_{\ell}$. STOPSS is not a scale-dependent value; if its value is $10^{-5}$, this criteria will be met when the first five digits of the residual sum of squares are the same at two successive iterations regardless of the size of the residual sum of squares.

The default value is approximately the maximum of $10^{-10}$ and $10^{-2} \cdot$ DIGITS/3, where DIGITS is the number of decimal digits carried by the user's computer for a single precision value when the single precision version of STARPAC is being used, and is the number carried for a double precision value otherwise. When the user-supplied value for STOPSS is outside the interval [10-DIGITS, 0.1], or when STOPSS is not an argument of the subroutine CALL statement, the default value will be used. 
--- The vector of dimension at least NPAR that contains the relative step sizes used to approximate the derivative matrix numerically. It is input to the estimation subroutines and returned from the step size selection subroutines. The procedure used to select the default values is described in $\S E .2$. For the estimation subroutines, when STP is not an argument of the subroutine CALL statement or when $\operatorname{STP}(1) \leqslant 0$, the default values will be used for a 11 of the step sizes, and when $\operatorname{STP}(1)>0$, values of $\operatorname{STP}(k) \leqslant 0$, $k=2, \ldots$, NPAR, will be interpreted as an input error.

VCV <-- The matrix of dimension at least NPARE by NPARE that contains the variance-covariance matrix of the estimated parameters, approximated as designated by argument IVAPRX. The parameters which are held fixed [see argument IFIXED] are not included in the variance-covariance matrix.

The approximation of the variance-covariance matrix is based on a linearization of the model in the neighborhood of the solution; the validity of the approximation depends on the nonlinearity of the model. This approximation may be extremely inaccurate for a problem with a highly nonlinear model.

WT $\rightarrow$ The vector of dimension at least $N$ that contains the weights. Negative weights are not allowed and the number of nonzero weights must equal or exceed the number of parameters being estimated. A zero weight eliminates the corresponding observation from the analysis, although the predicted values and standard deviations of the predicted values are still computed. [See Appendix A.]

XM $\quad->$ The matrix of dimension at least $N$ by $M$ whose $j^{\text {th }}$ column contains the $N$ values of the $j^{\text {th }}$ independent variable, $j=1, \ldots, M$.

$\mathrm{Y} \quad \rightarrow$ The vector of dimension at least $\mathrm{N}$ that contains the dependent variable.

E. Computationa1 Methods

E.1 Nonlinear Least Squares Estimation

E.1.a The Nonlinear Least Squares Algorithm

The nonlinear least squares estimation subroutines use the NL2SOL software package written by Dennis et a1. [1981a and 1981b]. The observations of the dependent variable, which are measured with error, are iteratively fit to a nonlinear model by minimizing the sums of squares of the errors as described in $\$ A$. The iterations continue until the convergence criteria based on the change in the parameter values or in the residual sum of squares are satisfied [see $\S D$, arguments STOPP and STOPSS], the maximum number of iterations (or model subroutine calls) is reached [see §D, argument MIT], or the iterations are terminated due to singularity in the model or false convergence. All but the first of these stopping conditions may indicate computational problems and will produce an error report. [See Donaldson and Tryon, 1983, §D.5.] 
Singular convergence means that the model contains too many parameters, at least near the solution, while false convergence can indicate that either STOPSS or STOPP is set too small for the accuracy to which the model and its derivatives are being computed, or that there is an error or discontinuity in the derivative. Users should examine their models to determine and correct the underlying cause of singular or false convergence.

Iterative procedures for solving nonlinear least squares problems are discussed in Dennis and Schnabel [1983], Draper and Smith [1981], and Kennedy and Gentle [1980]. The specific procedure used in STARPAC is as follows. At iteration $\ell+1$ the values of the parameter vector $\beta_{\ell+1}$ are given by

$$
B_{\ell+1}=B_{\ell}-\left(D_{\ell}{ }^{T} \cdot W \cdot D_{\ell}+S_{\ell}+\Lambda_{\ell}\right)^{-l} \cdot D_{\ell}{ }^{T} \cdot W \cdot \varepsilon_{\ell}{ }^{T}
$$

subject to the restriction that

$$
\left\{\sum_{k=1}^{\operatorname{NPAR}}\left[\left(\beta_{\ell+1}(k)-\beta_{\ell}(k)\right) / \operatorname{SCALE}(k)\right]^{2}\right\}^{1 / 2} \leqslant \delta_{\ell},
$$

where

NPAR is the number of parameters.

$B_{\ell} \quad$ is the vector of NPAR estimated parameter values from the $\ell^{\text {th }}$ iteration.

$\mathrm{D}_{\ell} \quad$ is the $\mathrm{N}$ by NPAR matrix of the partial derivatives,

$D_{\ell}(i, k)=\partial f_{i}\left(x_{i}, \beta_{\ell}\right) / \partial \beta_{\ell}(k) \quad$ for $i=1, \ldots, N$

and $k=1, \ldots$, NPAR.

$W$ is an $N$ by $N$ diagonal matrix of user-supplied weights, $W=\operatorname{diag}\left\{w t_{i}, i=1, \ldots, N\right\}$, when a weighted analysis is performed, and is the identity matrix otherwise.

$S_{\ell} \quad$ is an approximation to the exact term $S_{\ell}{ }^{*}$ in the matrix of second order terms (Hessian) of the Taylor series expansion of the residual sum of squares function,

$S_{\ell}{ }^{*}(j, k)=\sum_{i=1}^{N}\left[\varepsilon_{\ell} \cdot w t_{i} \cdot\left(\partial^{2} \varepsilon_{\ell}(i) / \partial \beta_{\ell}(j) \partial \beta_{\ell}(k)\right)\right]$,

for $j=1, \ldots$, NPAR, and $k=1, \ldots$, NPAR.

$\varepsilon_{\ell} \quad$ is the vector of the $N$ residuals from the previous iteration.

SCALE is the vector of the scale value (i.e., the typical size) of each of the NPAR parameters. They are either selected by STARPAC or are supplied by the user via subroutine argument SCALE [see §D]. 
$\delta_{\ell} \quad$ is the adaptively chosen diameter of the trust region, i.e., the region in which the local approximation to the user's model function is reliable. At each iteration $\ell, \delta_{\ell}$ is computed based on information from the previous iteration. At the first iteration, the initial value, $\delta_{0}$, is supplied by argument DELTA [see $\$ D$ ], which can be used to control the change in the parameters permitted at the first iteration.

$\Lambda_{\ell} \quad$ is an NPAR by NPAR diagonal matrix, $\Lambda_{\ell}=\operatorname{diag}\left\{\lambda_{\ell} / \operatorname{SCALE}(k), k=1, \ldots\right.$, NPAR\}, where $\lambda_{\ell}$ is chosen to approximate the smallest non-negative number such that the restriction given above on the size of the change in the parameters is satisfied.

The second order term $S_{\ell}{ }^{*}$, which is expensive and difficult to compute accurately, is important only if it is large compared to the term $D_{\ell}{ }^{T} \cdot W \cdot D_{\ell}$, that is, when the residuals are large or the model is highly nonlinear. When $\mathrm{S}_{\ell}{ }^{*}$ is large compared to $\mathrm{D}_{\ell}^{\mathrm{T}} \cdot \mathrm{W} \cdot \mathrm{D}_{\ell}$, algorithms which ignore it, such as Levenberg-Marquardt or Gauss-Newton, may converge slowly. The NL2SOL algorithm used by STARPAC, however, adaptively decides when inclusion of this term is necessary for reliable results and uses an inexpensive approximation to $S_{\ell}{ }^{*}$ in those cases.

The matrix, D, of partial derivatives of the model with respect to each parameter is either computed analytically using a user-supplied subroutine [see $\S D$, argument NLSDRV], or is numerically approximated using forward difference quotients as described in $\S \mathrm{E} .2$. When the derivatives are approximated numerically, the least squares solution, especially the variance-covariance matrix, can be sensitive to the step sizes used for the approximation. The user may want to use STARPAC subroutines STPLS or STPLSC to recompute the step sizes at the solution provided by the estimation subroutines to assure that the step sizes which were used are still acceptable. If there is a significant change in the step size, the least squares solution should be recomputed with the new step sizes from the current point. In addition, if the estimation subroutine has convergence problems, the user may want to recompute the step sizes with the most recent parameter values to see if a change in the curvature of the model, which will be reflected as a change in the optimum step sizes, is causing the problem.

Dennis et a1. [1981a] provides a detailed description of the algorithm used in STARPAC. STARPAC also includes the subroutines NL2SOL, NL2SNO, and NL2ITR, which they reference, and which can be used as documented by them. [See Dennis et a1., 1981b.]

\section{E.1.b Computed Results and Printed Output From the Nonlinear Least Squares Regression Subroutines}

The argument controlling the printed output, NPRT, is discussed in $\S D$. The output from the nonlinear least squares estimation subroutines consists of five sections, several of which include tables summarizing the results. In the following descriptions, the actual table headings are given by the underlined, uppercase phrases. Results which correspond to input or returned subroutine CALL statement arguments are identified by the argument name in upper case (not underlined). 
Section 1 provides a summary of the initial estimates and control values. It lists the following information.

- The initial values of the parameters, PAR, and whether they are to be held fixed or not, IFIXED.

- The scale values, SCALE.

- Either the step sizes used to approximate the derivatives numerically, or, when user-supplied (analytic) derivatives are used, the results of the checking procedure; and the control values used in these computations as applicable [see $\S \mathrm{E} \cdot 2 \cdot \mathrm{b}$ and $\S \mathrm{E} .3 . \mathrm{b}]$.

- The number of observations, N.

- The number of observations with nonzero weights, NNZW.

- The number of independent variables, M.

- The maximum number of iterations allowed, MIT.

- The maximum number of model subroutine calls allowed.

- The two convergence criteria, STOPSS and STOPP.

a The maximum change in the parameters allowed at the first iteration, DELTA.

- The residual sum of squares computed using the starting parameter values.

- The residual standard deviation computed using the starting parameter values, RSD.

Section 2, lists selected information about each iteration and includes the reason the iterations were terminated. The information provided for each iteration includes the following.

- The iteration number.

- MODEL CALLS: the total number of times since execution began that the user's model subroutine has been called, not including calls required to approximate the derivatives numerically.

- RSD: the residual standard deviation computed using the parameter values from the current iteration.

- RSS: the residual sum of squares computed using the parameter values from the current iteration. 
- REL CHNG RSS: the relative change in the residual sum of squares caused by the current iteration.

- FORECASTED REL CHNG RSS: the forecasted relative change in the residual sum of squares at the current iteration, and whether this value was checked against STOPSS $(\underline{\operatorname{CHKD}}=\mathrm{Y})$ or $\operatorname{not}(\underline{\mathrm{CHKD}}=\mathrm{N})$.

- REL CHNG PAR: the maximum scaled relative change in the parameters at the current iteration, and whether this value was checked against STOPP $(\underline{\mathrm{CHKD}}=\mathrm{Y})$ or not $(\underline{\mathrm{CHKD}}=\mathrm{N})$.

- CURRENT PARAMETER VALUES: the estimated parameter values resulting from the current iteration.

Section 3 provides the following information for each observation, $i=1, \ldots, N$, based on the final solution.

- ROW: the row number of the observations.

- PREDICTOR VALUFS: the values for up to the first three columns of the independent variable matrix, XM, not including the first column if it is constant.

- DEPENDENT VARIABLE: the values of the dependent variable, Y.

- PREDICTED VALUE: the estimated predicted values, PV, from the fit.

- STD DEV OF PRED VALUE: the standard deviations of the predicted values, SDPV.

- RESIDUAL: the error estimates, RES.

- STD RES: the standardized residuals, SDRES.

- WEIGHT: the user-supplied weights, WT, printed only when weighted analysis is performed.

Section 4, displays the following plots of the standardized residuals.

- The standardized residuals versus row numbers.

- The standardized residuals versus predicted values.

- The standardized residuals versus the standardized residuals 1 agged by one observation, that $i s, \operatorname{SDRES}(i)$ versus $\operatorname{SDRES}(i-1), i=2 \ldots, N$.

- The normal probability plot of the standardized residuals.

Section 5, summarizes the following information about the final parameter estimates and their variances. 
- The variance-covariance matrix, VCV, of the estimated (unfixed) parameters, and the corresponding correlation matrix,

$$
r_{j k}=\operatorname{VCV}(j, k) /(\operatorname{VCV}(j, j) \operatorname{VCV}(k, k))^{1 / 2} \quad \begin{aligned}
& \text { for } j=1, \ldots, \text { NPARE } \\
& \text { and } k=1, \ldots, \text { NPARE. }
\end{aligned}
$$

- PARAMETER: the final value of each parameter, $\operatorname{PAR}(k), k=1, \ldots$, NPAR.

- SD OF PAR: the standard deviation of each estimated parameter, $(\operatorname{VCV}(k, k))^{1 / 2} \quad$ for $k=1, \ldots, N P A R$.

- RATIO: the ratio of each estimated parameter to its standard deviation, $\operatorname{RATIO}_{k}=\operatorname{PAR}(k) /(\operatorname{VCV}(k, k))^{1 / 2}$ for $k=1, \ldots$, NPAR.

- APPROXIMATE 95 PERCENT CONFIDENCE LIMITS: the lower and upper 95 percent confidence limits for each parameter, computed using the appropriate value of the Student's $t$ distribution with NNZW-NPARE degrees of freedom.

- the residual sum of squares, $\operatorname{RSS}(\beta)$.

- the residual standard deviation at the solution, RSD.

- the residual degrees of freedom, NNZW-NPARE.

- an approximation to the condition number of the derivative matrix, D (the Jacobian), under the assumption that the absolute error in each column of $D$ is roughly equal. The approximation will be meaningless if this assumption is not valid; otherwise it will usually underestimate the actual condition number by a factor of from 2 to 10 [see Dongarra et a1., 1979, p. 9.5].

NOTE: The standard deviation of the predicted values, the standardized residuals, the variance-covariance matrix, the standard deviations of the parameters, and the 95 percent confidence limits on the parameters are all based on a linear approximation to the model in a neighborhood of the solution; the validity of this approximation depends on the nonlinearity of the model. The statistics based on this approximation may be extremely inaccurate for a problem with a highly nonlinear model.

\section{E.2 Step Size Selection}

\section{E.2.a The Step Size Selection Algorithm}

The STARPAC step size selection subroutines use an algorithm developed by Schnabel [1982] to compute optimum step sizes for approximating the partial derivatives of the model with respect to each parameter. Briefly, the 
relative step sizes selected by these subroutines are those which produce forward difference quotient approximations to the derivative, $D_{f d}$, that agree reasonably well with the central difference quotient approximations, $D_{c d}{ }^{*}$ The central difference quotient approximations are twice as accurate but also twice as expensive to compute. Since the additional accuracy is not usually needed, central difference quotient approximations are not used by the estimation subroutines.

The number of reliable digits in these derivatives is a function of the step sizes used to compute them. Given properly chosen step sizes, the number of reliable digits in $D_{f d}$ and $D_{c d}$ will be approximately $n / 2$ and $n$, respectively, where $\eta$ is the number of reliable digits in the predicted values, PV, from the user's model subroutine. For example, if the predicted values are computed using an iterative procedure (such as quadrature or a solution of partial differential equations) which is expected to provide five good digits, then $n$ would be five; if the predicted values are calculated from a simple algebraic expression translated directly into Fortran code, then $n$ would (usually) be the number of decimal digits carried by the user's computer for the results.

The relative step size for $\beta(k), k=1, \ldots$, NPAR, is initially

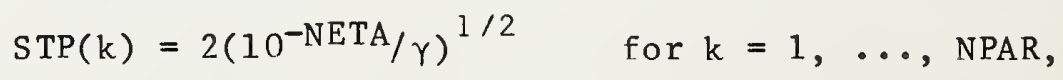

where

$\gamma$ is the average curvature (estimated by STARPAC) of the model with respect to $\beta(k)$.

NETA is the argument used to specify the number of reliable digits, $n$, in the model results.

The forward difference quotient approximations with respect to $\beta(k)$, $k=1, \ldots$, NPAR are then

$$
D_{f d}(i, k)=\frac{f_{i}\left(x_{i}, \beta^{k}\right)-f_{i}\left(x_{i}, \beta\right)}{\operatorname{sTP}(k) \cdot \operatorname{SCALE}(k) \cdot \operatorname{SIGN}(\beta(k))} \quad \text { for } i=1, \ldots, N \text {, }
$$

where

$\mathrm{N} \quad$ is the number of observations.

$f_{i} \quad$ is the function which models the $i^{\text {th }}$ observation.

$x_{i} \quad$ is the vector of the values of the $M$ independent variables at the $i^{\text {th }}$ observation.

B is the vector of the NPAR parameter values. 
is a vector which has the same values as $\beta$, except that the
$\mathrm{k}$ th parameter is equal to $\beta(k)+(\operatorname{STP}(k) \cdot \operatorname{SCALE}(k) \cdot \operatorname{SIGN}(\beta(k))) \cdot$

SCALE is the vector of the scale value (i.e., of the typical size) of each of the parameters.

SIGN is a function which returns the sign of its argument.

The central difference approximations to the model derivative with respect to $\beta(k), k=1, \ldots, N P A R$, are

$$
D_{c d}(i, k)=\frac{f_{i}\left(x_{i}, \beta^{+k}\right)-f_{i}\left(x_{i}, \beta^{-k}\right)}{3^{1 / 3} \cdot 10^{-N E T A / 3} \cdot \operatorname{SCALE}(k) \cdot \operatorname{SIGN}(\beta(k))} \text { for } i=1, \ldots, N \text {, }
$$

where

$\beta^{+k}$ is a vector which has the same values as $\beta$, except that the $k^{\text {th }}$ parameter is equal to

$$
B(k)+\left(3^{1 / 3} \cdot 10^{-N E T A / 3} \cdot \operatorname{SCALE}(k) \cdot \operatorname{SIGN}(B(k))\right) \text {. }
$$

$\beta^{-k} \quad$ is a vector which has the same values as $\beta$, except that the $k^{\text {th }}$ parameter is equal to

$$
\beta(k)-\left(3^{1 / 3} \cdot 10^{-N E T A / 3} \cdot \operatorname{SCALE}(k) \cdot \operatorname{SIGN}(B(k))\right) \text {. }
$$

The relative step size is considered acceptable if, for at least $\mathrm{N}-\alpha$ observations,

$$
\left|D_{f d}(i, k)-D_{c d}(i, k)\right| \leqslant \min \left\{10^{-N E T A} / 4,10^{-2}\right\} \quad \text { for } i=1, \ldots, N,
$$

where $\alpha$ is the number of observations exempted from meeting the above acceptance criterion [see $\S D$, argument EXMPT]. If the step size is not acceptable, it is adjusted by factors of 10 until the condition is met, or until no further decrease in the number of failures can be made, although in no case will the selected relative step size be greater than 1.0 or less than $10^{-\mathrm{NETA}}$.

Note that the step size selection subroutines will return the selected step sizes even when the number of failures exceeds the allowed value; this condition will be noted by the value of IERR. The detalled printed output should always be examined for problems discovered by the step size selection subroutines. 


\section{E.2.b Computed Results and Printed Output From the Derivative Step Size Selection Subroutines}

The argument controlling the printed output, NPRT, is discussed in $\S D$.

The output from the step size selection subroutines consists of a summary of the input and control values and, for each parameter, the selected relative step size, the number of observations at which this step size failed the step size selection criteria, and the row numbers at which the failures occurred.

\section{E.3 Derivative Checking}

\section{E.3.a The Derivative Checking Algorithm}

The STARPAC derivative checking subroutines use an algorithm developed by Schnabel [1982] to determine the validity of the user-supplied derivative subroutine. The user-supplied derivative subroutine is considered correct for a given row $i, i=1, \ldots, N$, and coefficient $B(k), k=1, \ldots$, NPAR, if

$$
\left|D_{f d}(i, k)-D(i, k)\right| \leqslant 10^{-\tau}|D(i, k)|
$$

where

D is the derivative computed by the user's subroutine.

$D_{f d} \quad$ is the forward difference quotient approximation to the derivative. [See $§ E .2 . a \cdot]$

$\tau$ is the agreement tolerance, i.e., number of digits of agreement required between $D$ and $D_{f d}$, which must be less than or equal to the number of good digits in $\mathrm{D}_{\mathrm{fd}}$. [See $\S \mathrm{D}$, argument NTAU.]

When the agreement tolerance is not satisfied, the checking subroutine attempts to determine whether the disagreement is due to an error in the user's code or is due to the inaccuracy of the difference quotient approximation, caused either by high curvature in the user's model or by significant roundoff error.

The derivative checking subroutines each check only one row of the derivative matrix. The user should examine the row at which the derivatives were checked to ensure that some relation between the coefficients and independent variables, such as a zero-valued independent variable or a factor $\left(x_{i}-\beta(k)\right)$ when $x_{i}=\beta(k)$, is not hiding the effect of an incorrectly computed derivative. Checking only one row is appropriate since the same code is frequently used to compute the model function and derivatives at each row $i=1, \ldots, N$, as is the case in the examples shown in $\S \mathrm{F}$. If the code used to express the model function and derivatives is not the same for each row, then each distinct section of the code should be checked by making multiple calls to DCKLSC with NROW set to a row within each section. [See §D, argument NROW.] 


\section{E.3.b Computed Results and Printed Output \\ From the Derivative Checking Subroutines}

The argument controlling the printed output, NPRT, is discussed in $\S D$.

The output for the derivative checking subroutines consists of a summary of the input and control values, and the results of the derivative checking test with respect to each of the model parameters, $B(k), k=1, \ldots$, NPAR. The possible test results are:

OK -

- The user-supplied derivative and the numerical derivative agree to the required number of digits.

QUESTIONABLE -

- The user-supplied derivative and the approximated derivative agree to the required number of digits but both are equal to zero. The user should recheck the derivative at another row.

- The user-supplied derivative and the approximated derivative do not agree to the required number of digits, but the user-supplied derivative is identically zero and the approximated derivative is approximately zero. The user should recheck the derivative at another row.

- The user-supplied derivative and the approximated derivative disagree, but the user-supplied derivative is identically zero. The user should recheck the derivative at another row.

- The user-supplied derivative and the approximated derivative disagree, but the validity of the approximated derivative is questionable because either the ratio of the relative curvature of the model to the slope of the model is too high, or SCALE(k) is wrong.

- The user-supplied derivative and the approximated derivative disagree, but the validity of the estimated derivative is questionable because the ratio of the relative curvature of the model to the slope of the model is too high.

\section{INCORRECT -}

- The user-supplied derivative and the approximated derivative disagree, and there is no reason to question the accuracy of the approximated derivative. 


\section{F. Examples}

The sample programs of this section use the model and data given in example one, pages 428 to 441 of Daniel and Wood [1980]; the model is

$$
f_{i}\left(x_{i}, \beta\right)=\beta(1) \cdot x_{i}(1)^{\beta(2)}, \quad \text { for } i=1, \ldots, N \text {. }
$$

In the sample program of figure F-1a, NLS is used to compute the least squares solution using numerically approximated derivatives; figures $\mathrm{F}-1 \mathrm{~b}$ through F-lf show the output from NLS.

In sample program of figures $\mathrm{F}-2 \mathrm{a}$, NLSD is used to compute the least squares solution given analytic derivatives; figures $F-2 b$ through $F-2 f$ show the output from NLSD.

In the example program of figure F-3a, STPLS is used to compute the optimum step sizes for numerically approximating the derivatives with respect to each of the parameters, $\beta(k), k=1,2$. Figure $F-3 b$ shows the output from STPLS.

In the example program of figure F-4a, DCKLS is used to check the validity of a user-supplied derivative subroutine. In figure F-4a, the derivative subroutine has been intentionally coded incorrectly in order to display the report obtained when the derivative checking subroutine determines the derivatives are incorrect, and the starting parameter values have been chosen in order to display the report obtained when the test results are questionable. Figure $F-4 b$ shows the output from DCKLS. 
Nonlinear Least Squares Estimation

With Numerically Approximated Derivatives

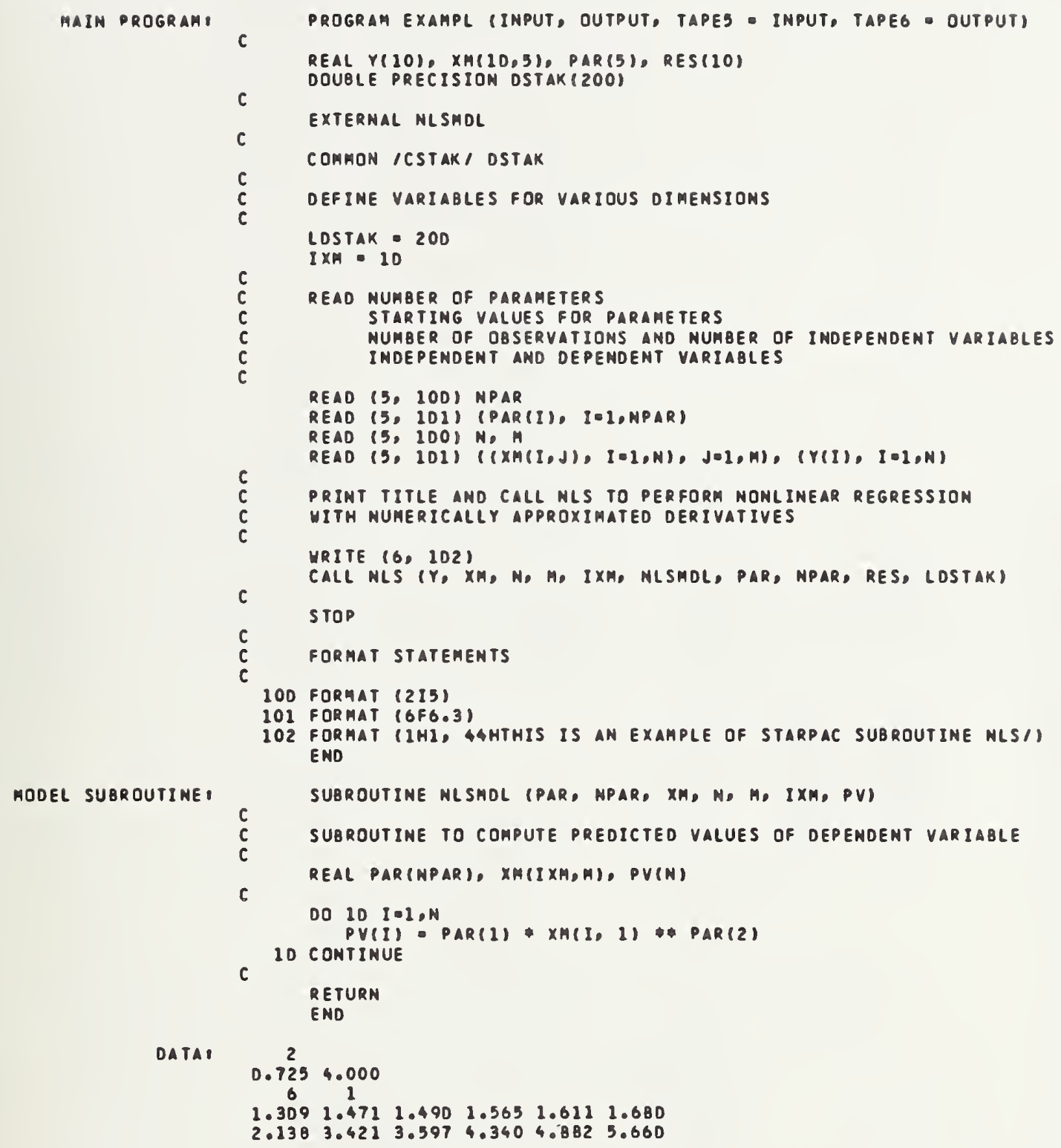

Figure $\mathrm{F}-1 \mathrm{a}$

Example program and data using NLS 


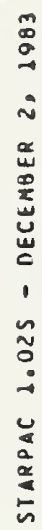

免

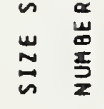

离

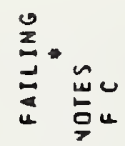

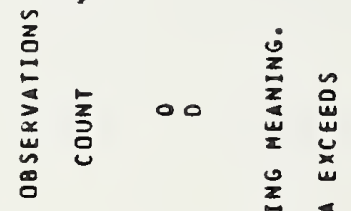

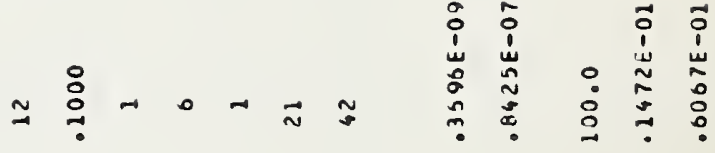

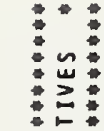

管

운

至

a

更

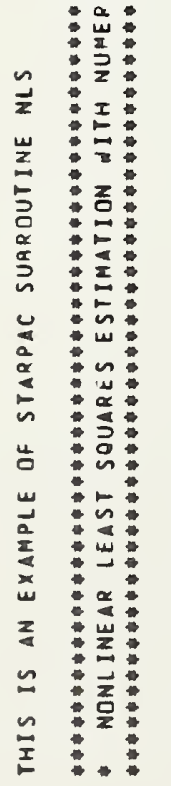

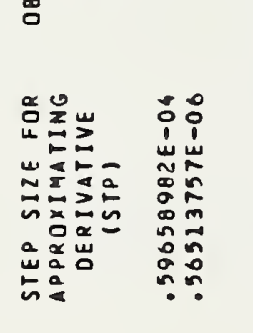

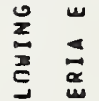

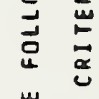

ธ

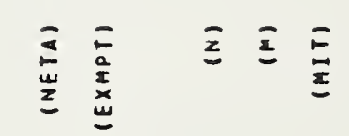

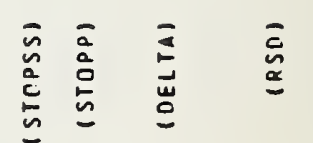

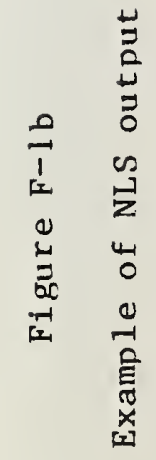

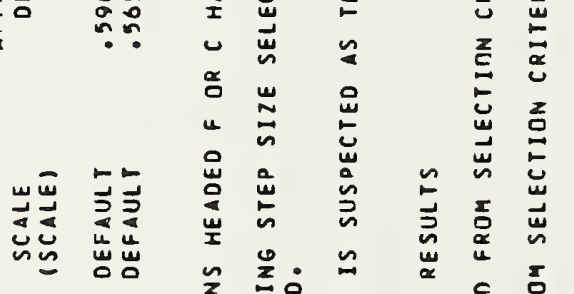

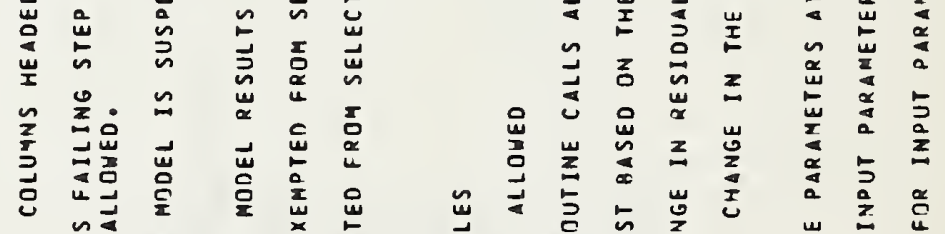

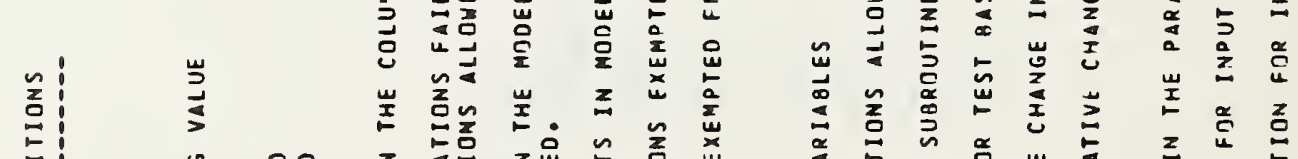

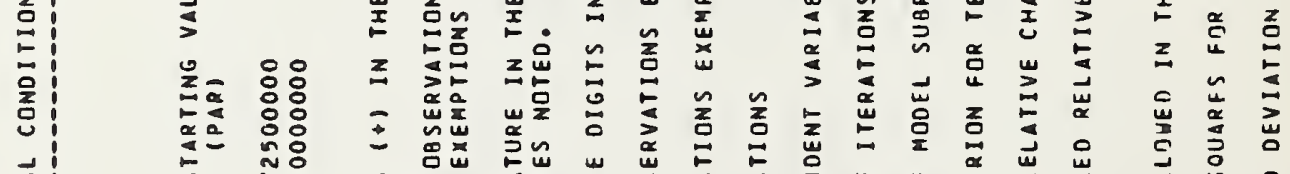

|

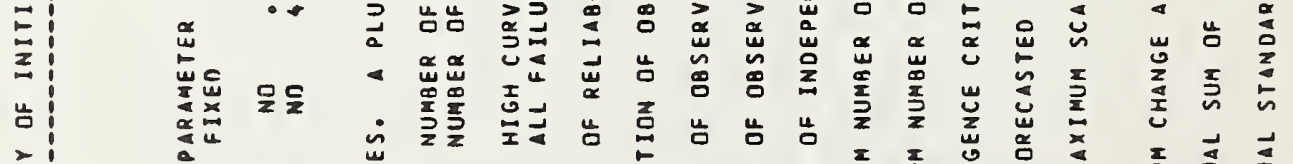

a

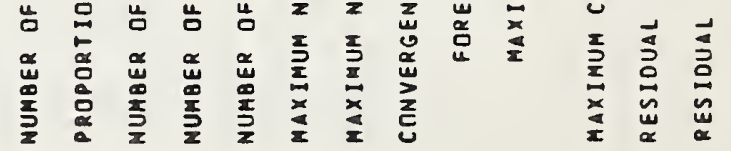




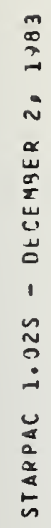

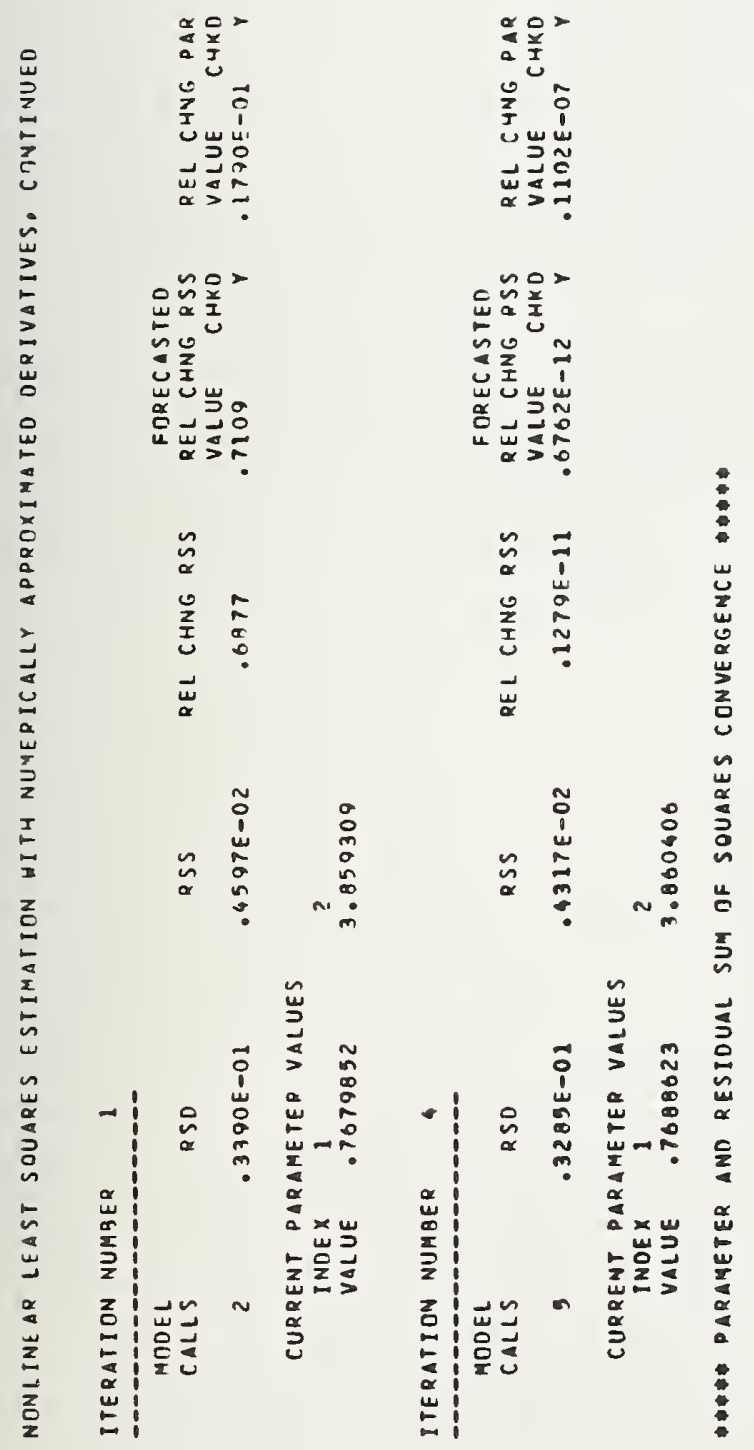




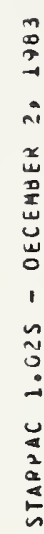

芯出蓄:

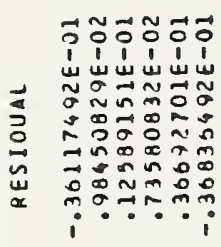

$\overrightarrow{0} \overrightarrow{0} \overrightarrow{0} \overrightarrow{0} \overrightarrow{0}$

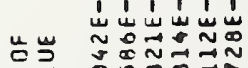

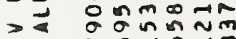

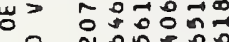

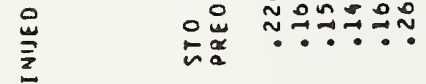

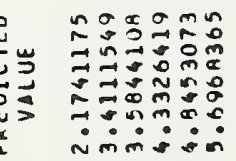

느

西

崖等

岁>

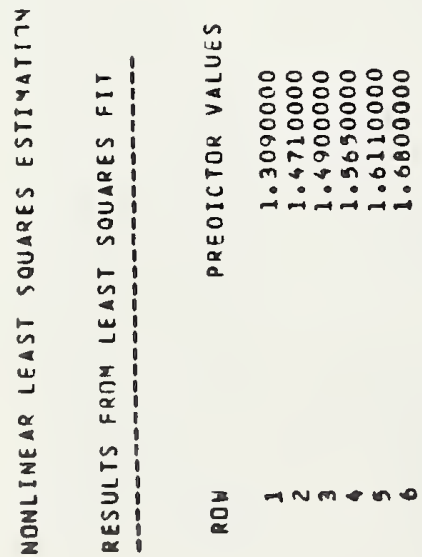




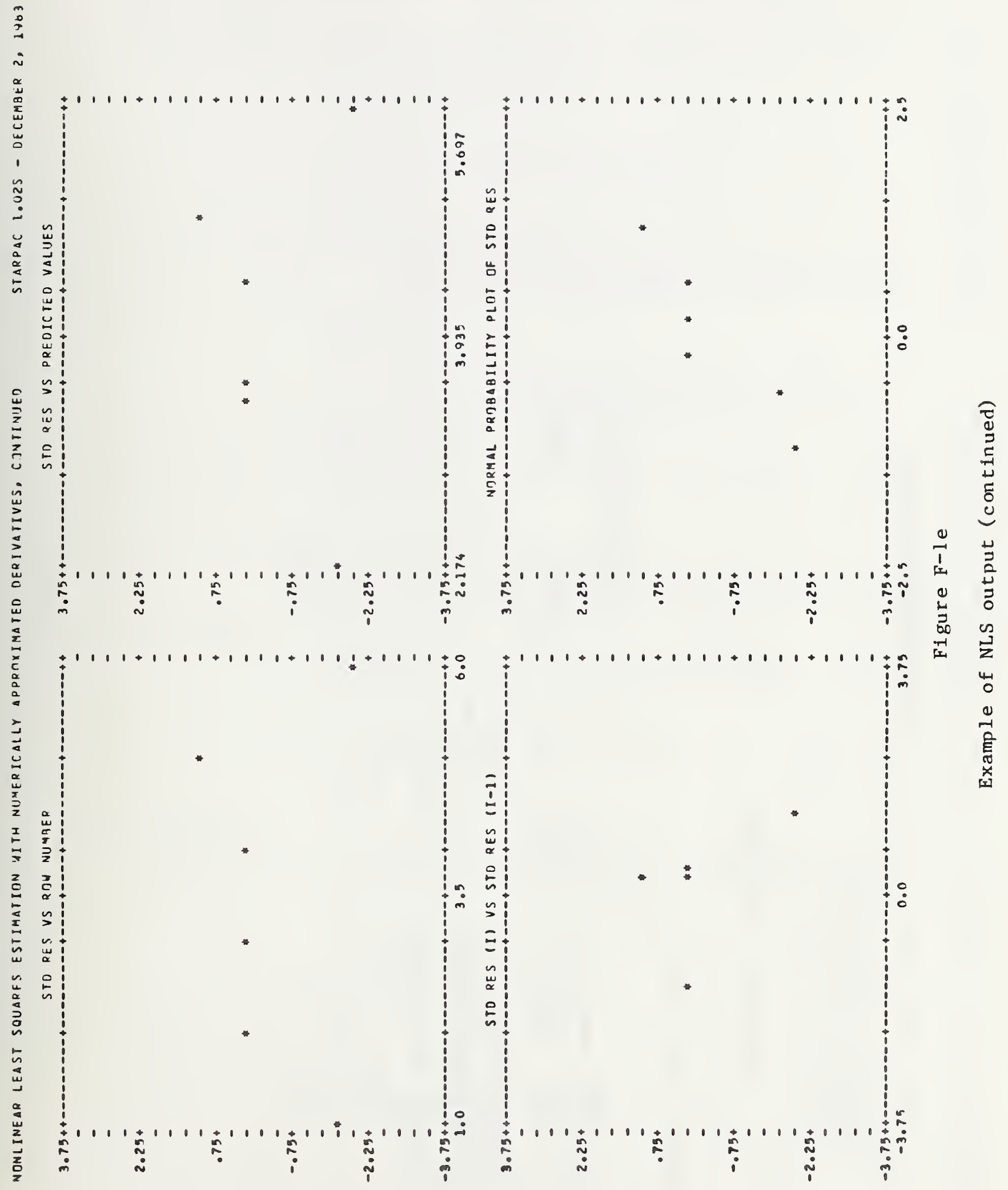




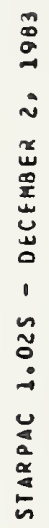

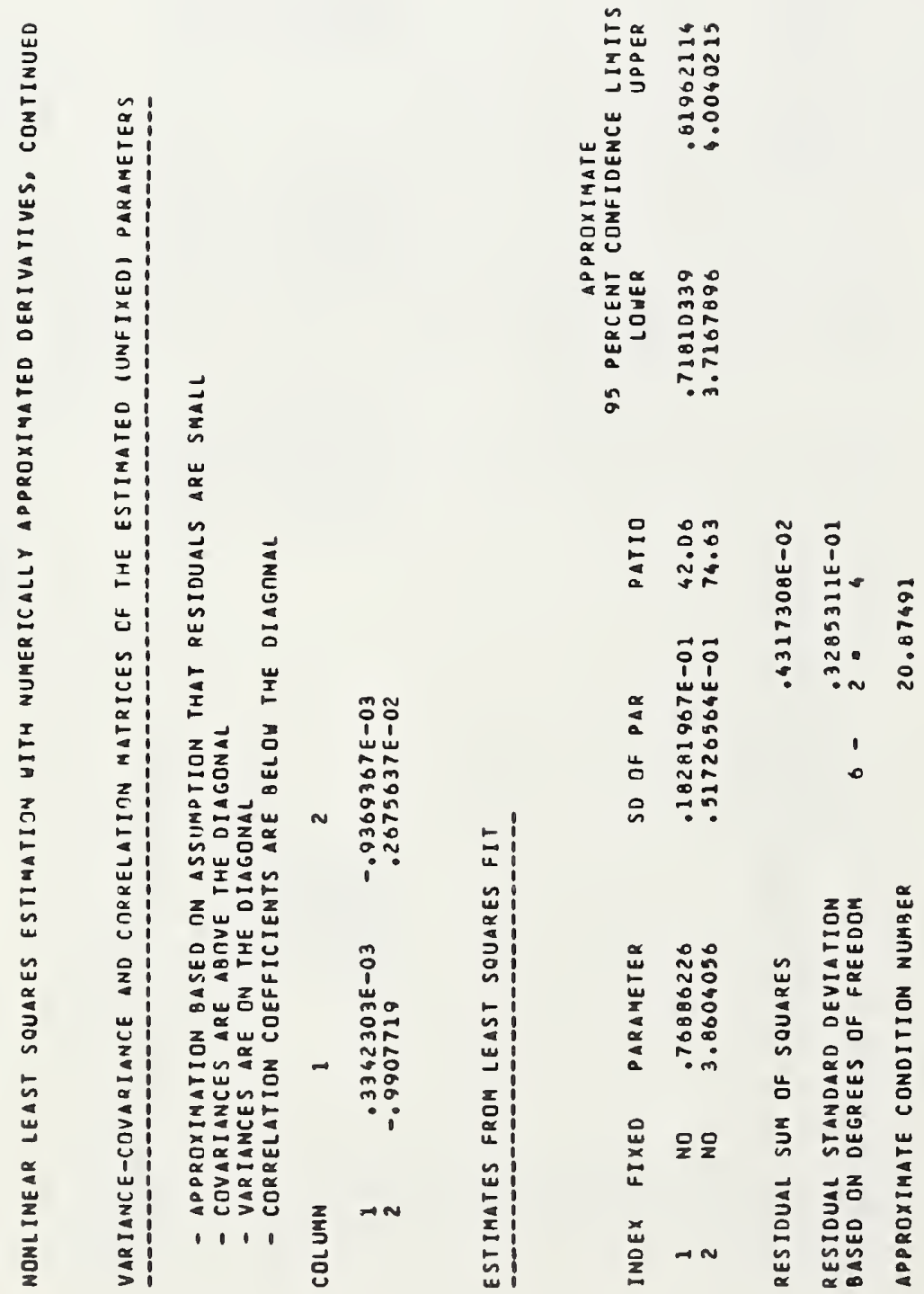


Nonlinear Least Squares Estimation

With User-Supplied Analytic Derivatives

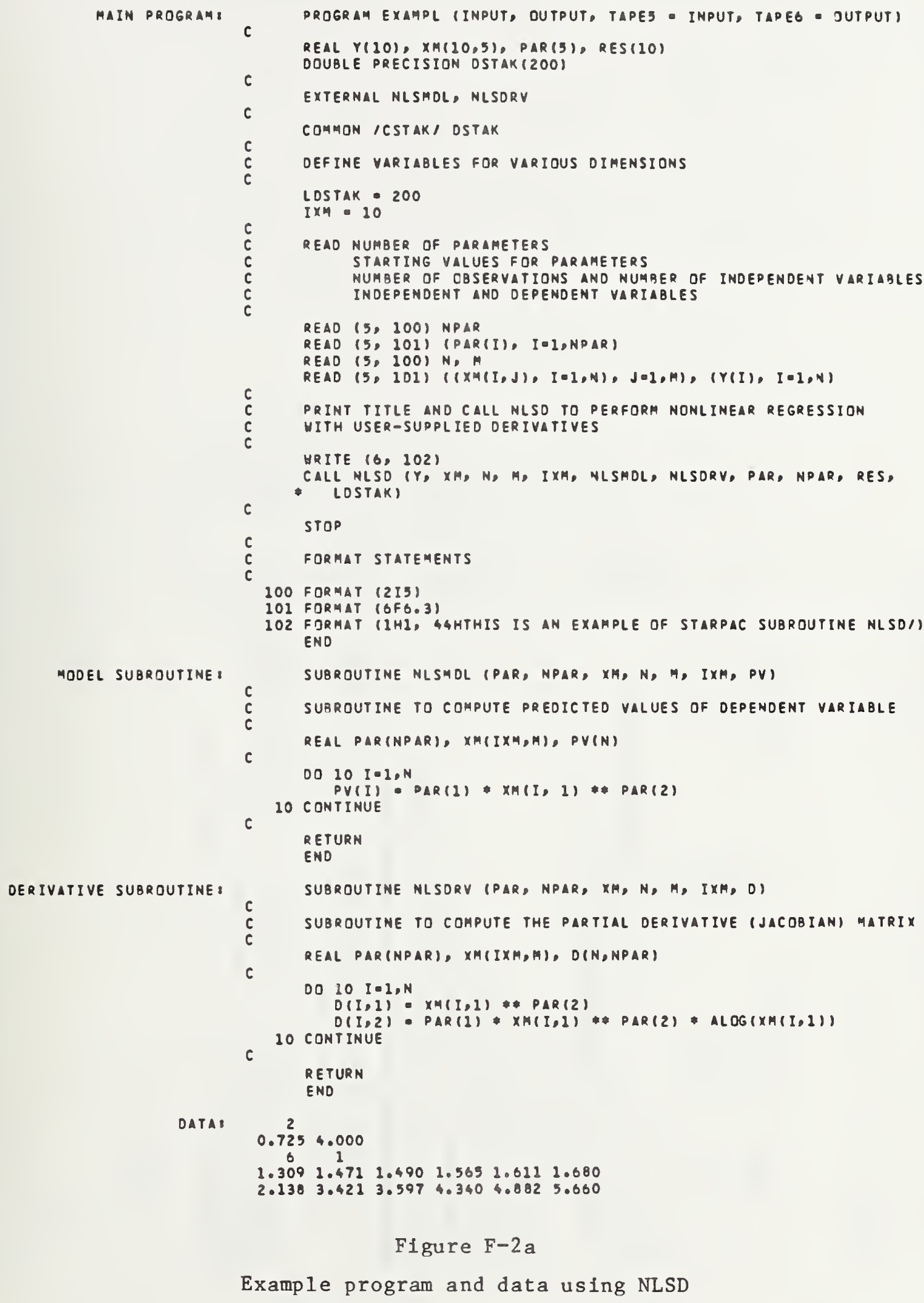$$
\begin{aligned}
& c \\
& c \\
& c \\
& c
\end{aligned}
$$

Example program and data using NLSD 


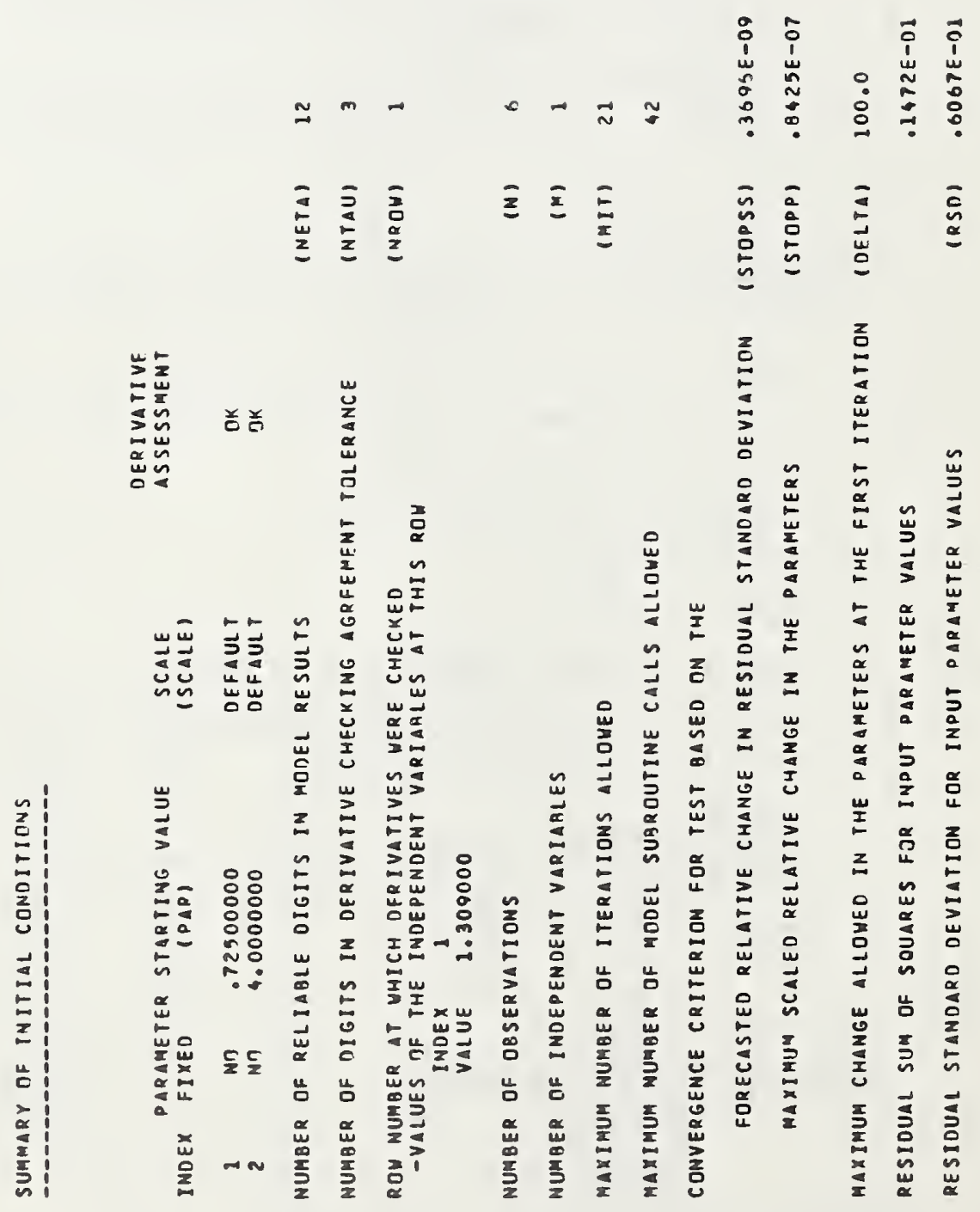




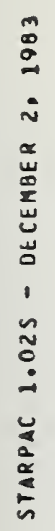

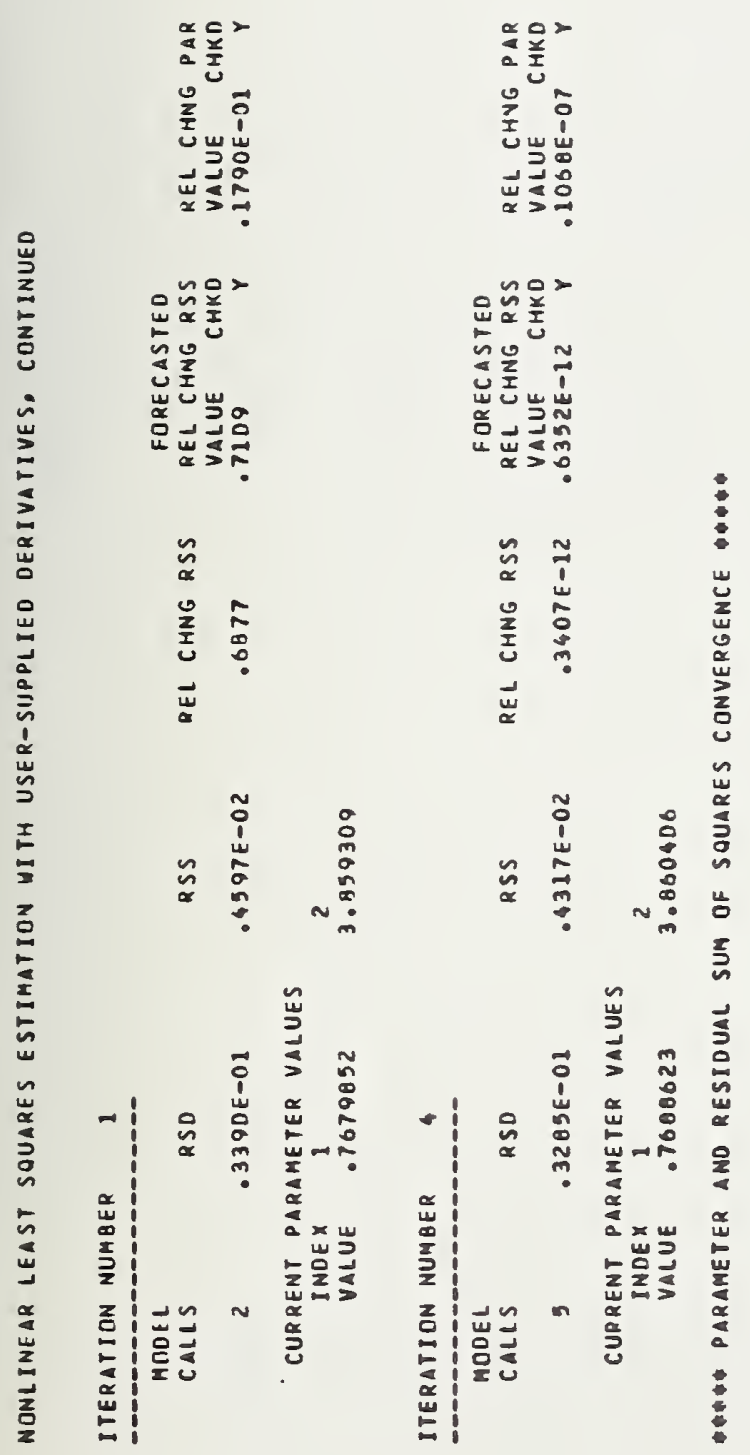




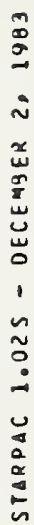

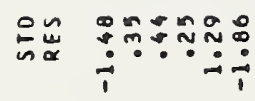

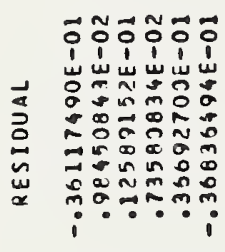

무유무우

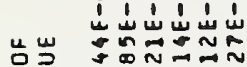

$>$ व

山

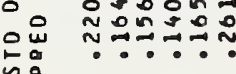

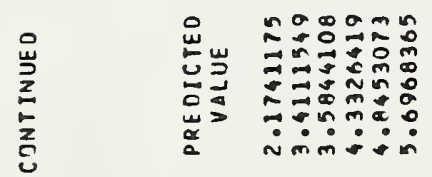

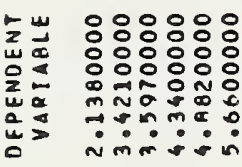

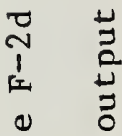

है क

II

4

告 


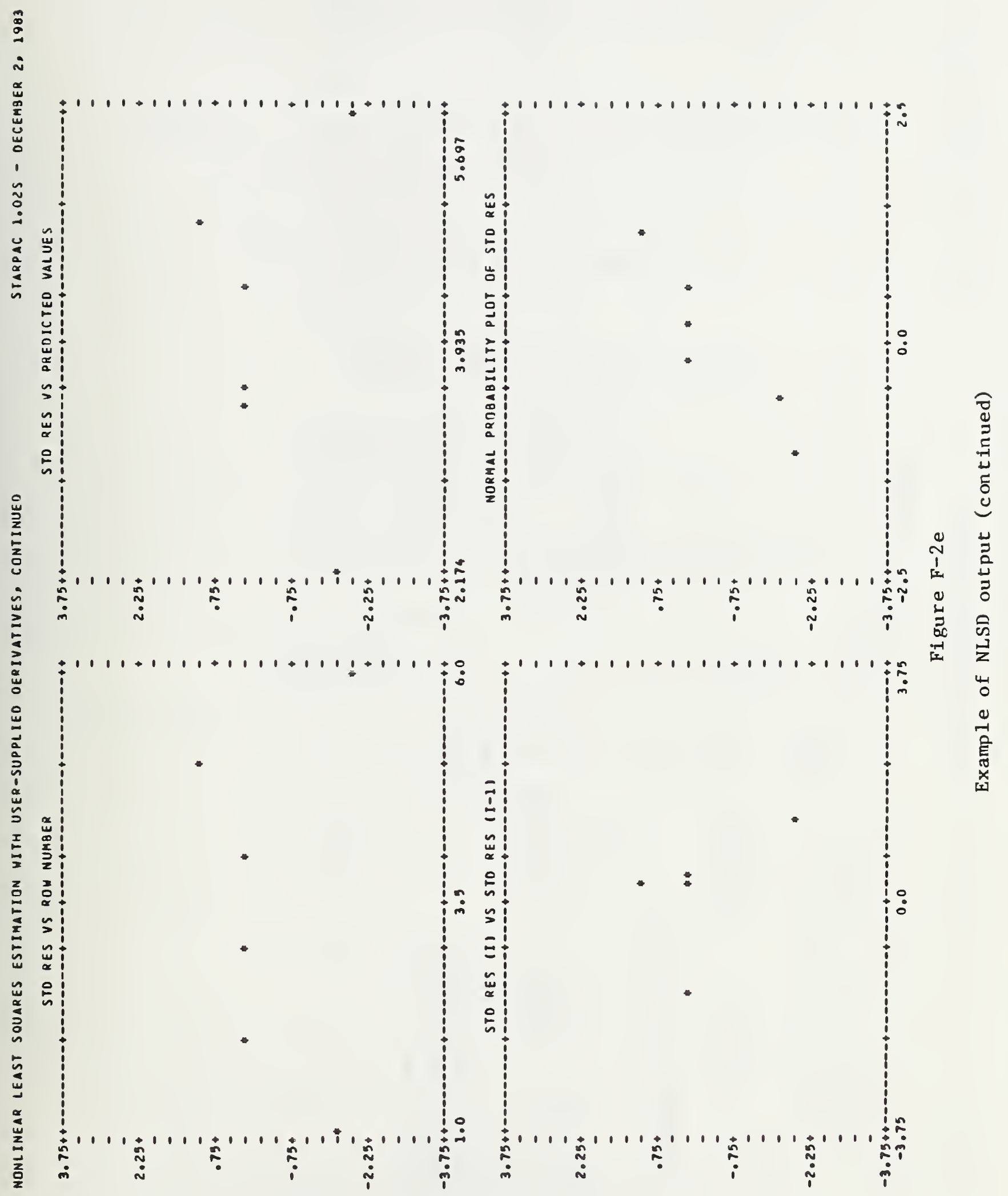




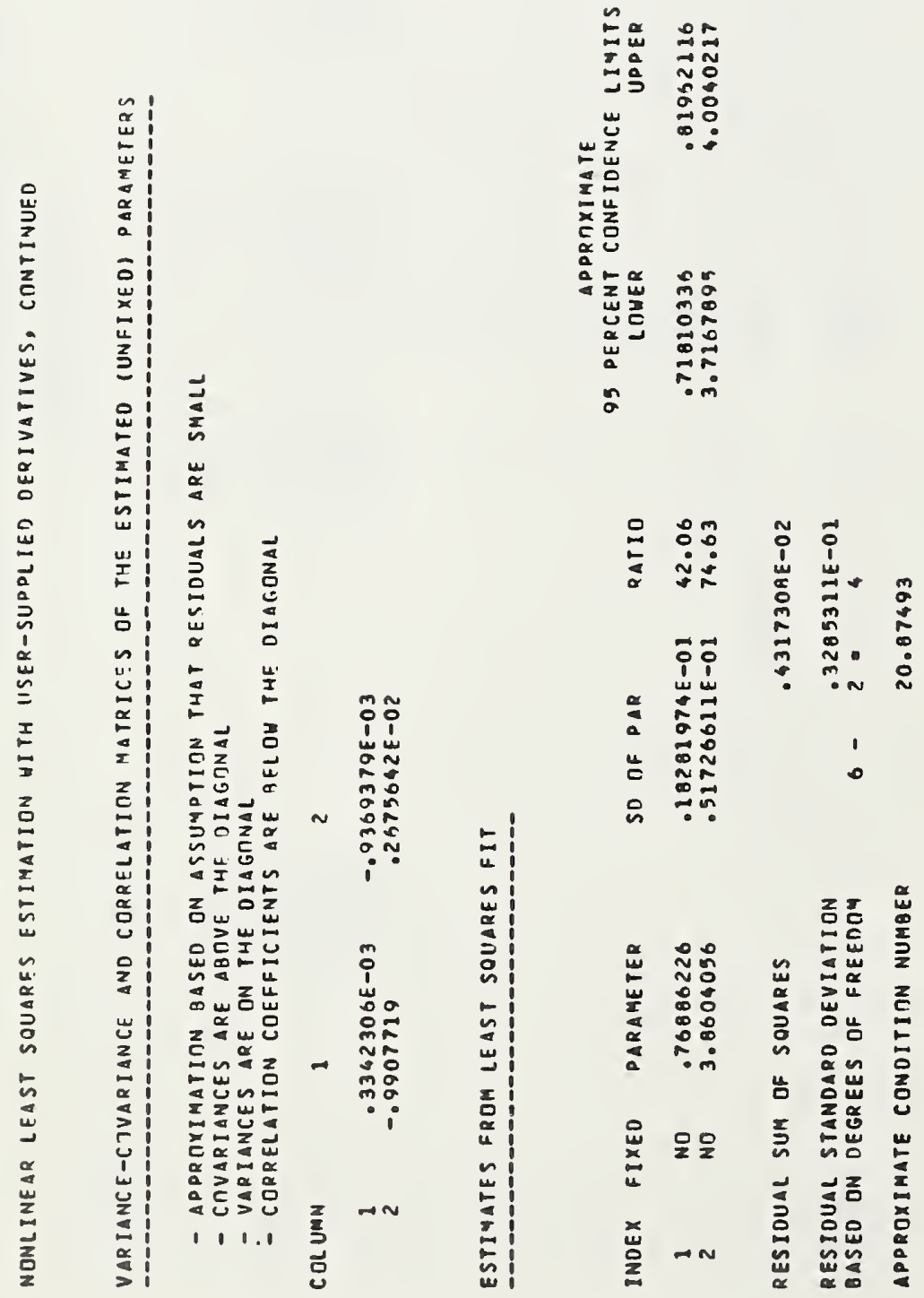




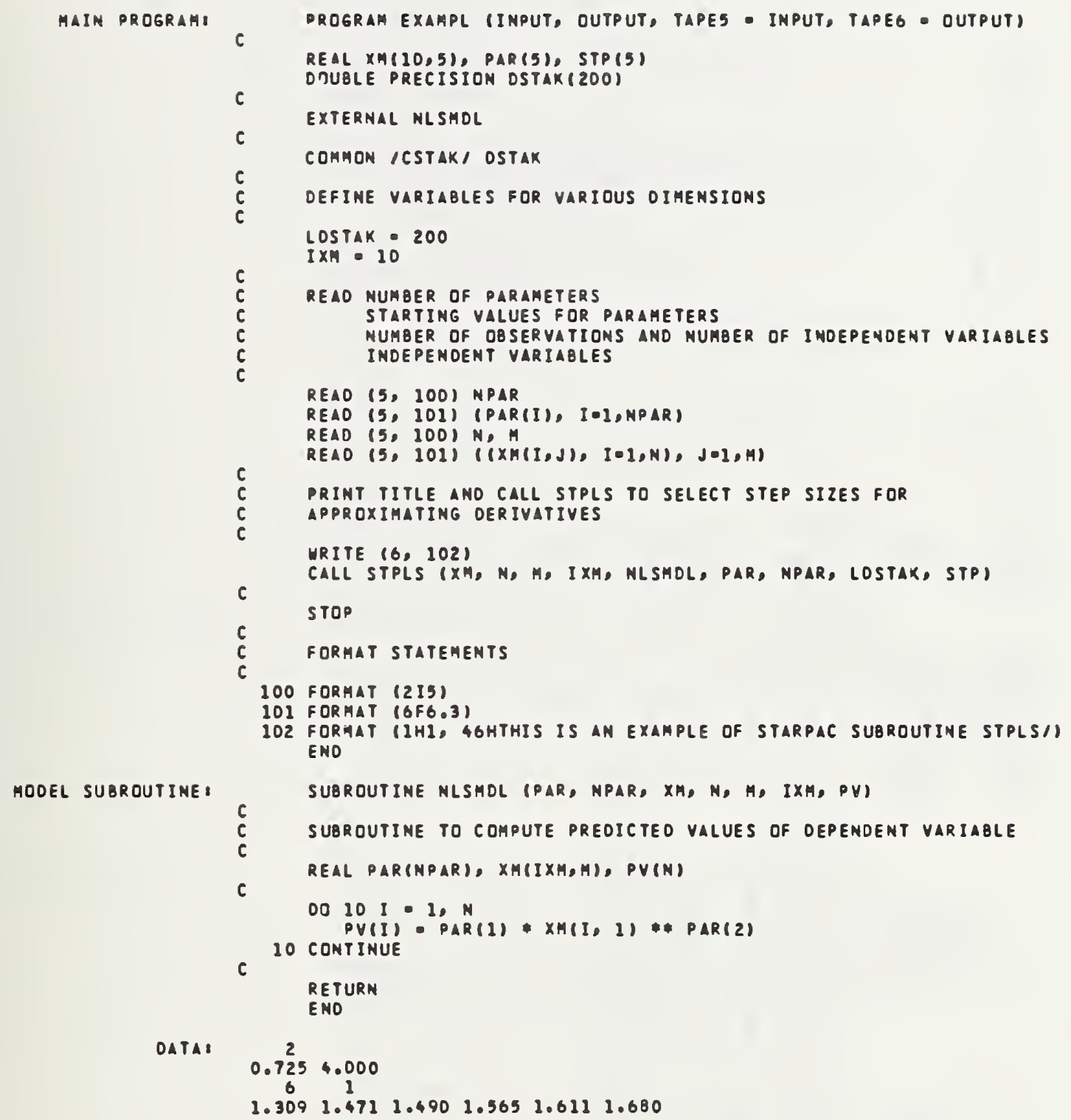

Example program and data using STPLS 


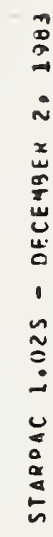

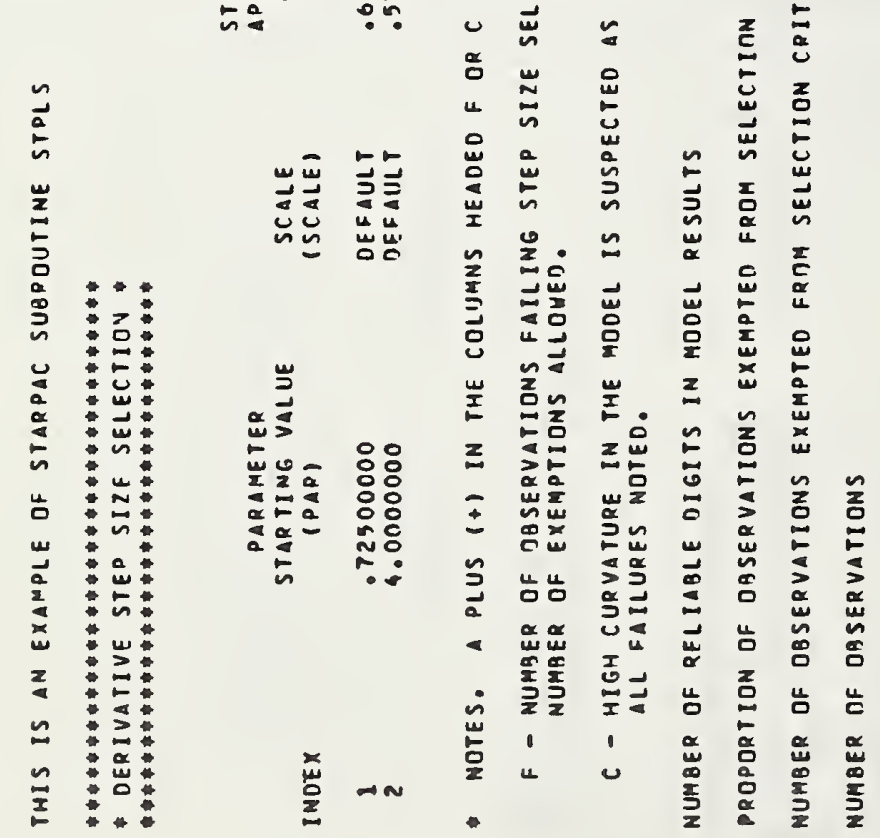




\section{Derivative Checking}

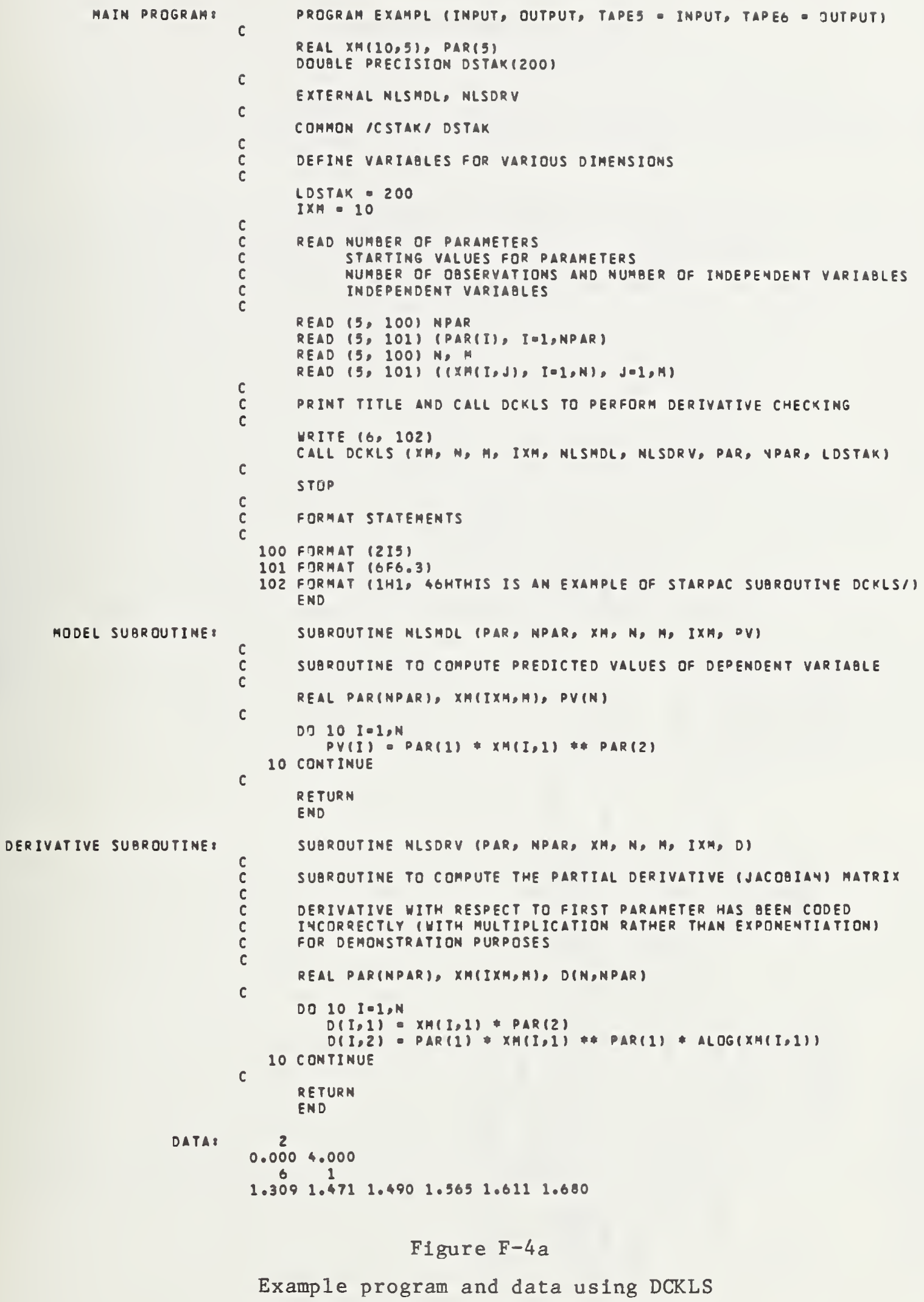

Figure $\mathrm{F}-4 \mathrm{a}$

Example program and data using DCKLS

$$
2-1.45
$$




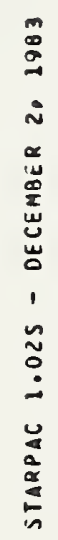

$\stackrel{\vec{x}}{u}$

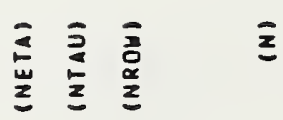

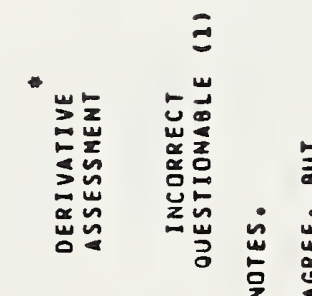

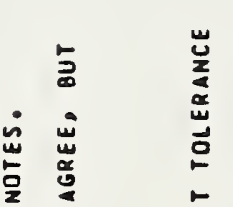

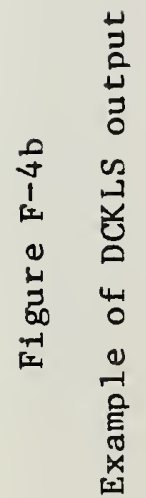

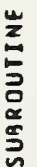

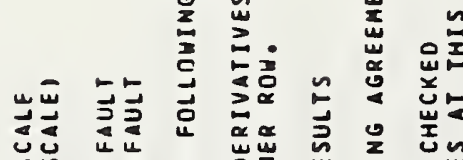

幽 嵌岀

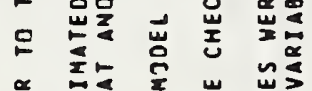

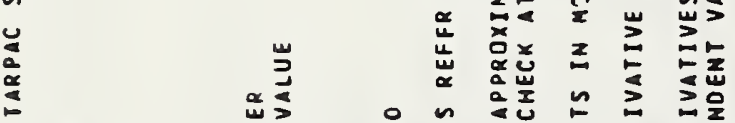

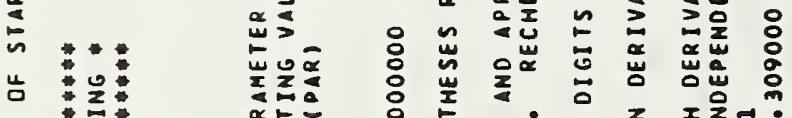

品少

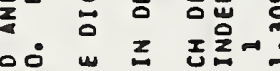

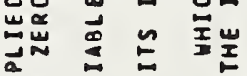

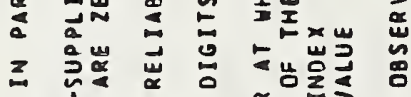

:

$z$

$\simeq 2$

$\cong$

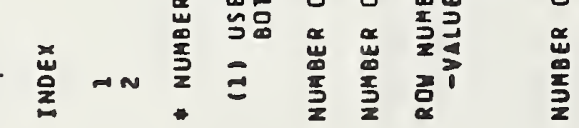


Appendix A

WEIGHTED LEAST SQUARES

Weighted least squares can be used to eliminate observations from the analysis, and to compensate for unequal variances in the observational errors.

Observations can be eliminated from the analysis by using weight values consisting only of zeros and ones. This will produce the same results as performing an unweighted analysis with the zero-weighted values removed except that the predicted values, the standard deviations of the predicted values, and the residuals of the zero-weighted data are computed. There are two main reasons for weighting observations zero. The first is to obtain the predicted values and their standard deviations for a set of independent variables not included in the observed data. (This is done by assigning any arbitrary value to the dependent variable of the desired set of independent variables, and then weighting these values zero.) The second reason is to allow easy examination of the effect of outliers and influential data points. Outliers often appear as large values in residual plots. Careful checking of the data often leads to confirmation that the data are in error, and sometimes to a correction. When a cause for suspicious data cannot be found, it may be advisable to compare the analysis with and without the questionable data. Caution is in order if the estimates or conclusions are highly sensitive to a small amount of suspicious data. Data that have a very high influence on a fitted curve may not result in large residuals, however, even if they are in error. In fact, extremely influential observations may force the fitted curve to be very close, leading to very small residuals. It is therefore desirable to identify influential observations and to compare the results obtained with and without these points. Several methods for detecting influential observations are discussed in Bement and Williams [1969], Cook [1977], Hoaglin and Welsch [1978], and Belsley et al. [1980].

Using weights to compensate for unequal observational error variances is not as straightforward as using zero weights to eliminate observations from the analysis. When the variances of the observational errors, $\varepsilon_{1}$, are not equal, the unweighted least squares estimates remain unbiased but do not have minimum variance. Minimum variance estimates are obtained by using weights $w t_{i}=1 /$ Variance $\left[\varepsilon_{i}\right]$ when the error variances are known. If weights must be estimated, they should be based on at least 10 degrees of freedom [see Bement and Williams, 1969]. In practice, however, weights are derived from theory, or obtained from the data being fit, and either of these methods can do more harm than good. When the need for weights is suspected and the error variances are not known, first fit the data using unweighted least squares; analysis of the residuals may confirm the need for weighting and may also provide estimates for the weights themselves. If the need for weights is confirmed, then a statistician should be consulted to assist in selecting the weights and in interpreting the results. 
The number of reliable digits, $n$, in the results of a real valued function, $g(\beta)$, can be estimated in most cases by evaluating

$$
n=-\log _{10}\left(\max _{j=-2, \ldots, 2} \frac{\left\{\lg \left(\beta^{j}\right)-[a+j \cdot b] \mid\right\}}{|g(\beta)|}\right.
$$

where

$\beta^{j} \quad$ is the vector of the NPAR parameters of the function given by,

$$
\beta^{j}(k)=\beta(k)+j \cdot 10^{-(\operatorname{DIGITS} / 2) \cdot \beta(k)} \quad \begin{aligned}
& \text { for } j=-2, \ldots, 2, \\
& \text { and } k=1, \ldots, \text { NPÁR, }
\end{aligned}
$$

where

DIGITS is the number of decimal digits carried by the user's computer for a single precision value when the single precision version of STARPAC is being used and is the number carried for a double precision value otherwise.

a

$=(0.20) \cdot \sum_{j=-2}^{2} g\left(\beta^{j}\right)$.

b

$$
=(0.10) \cdot \sum_{j=-2}^{2} j \cdot g\left(\beta^{j}\right) \text {. }
$$

This procedure may underestimate the number of reliable digits if $\mathrm{g}(\beta)$ is extremely nonlinear. A more elaborate and more robust procedure is described in Gill et al. [1981]. 
American National Standards Institute. (1966). ANS FORTRAN X3.9-1966. New York: American National Standards Institute.

Bard, Y. (1974). Nonlinear parameter estimation. New York: Academic Press.

Belsley, D.A.; Kuh, E.; Welsch, R.E. (1980). Regression diagnostics. New York: John Wiley and Sons.

Bement, T. R.; Williams, J. S. (1969). Variance of weighted regression estimators when sampling errors are independent and heteroscedastic. J. Amer. Statists. As soc. 64: 1369-1382.

Cook, D. R. (1977). Detection of influential observations in linear regression. Technometrics, 19: 15.

Danie1, C.; Wood, F. S. (1977). Fitting equations to data. New York: John Wiley and Sons.

Dennis, J. E. Jr.; Gay, D. M.; Welsch, R. E. (1981a). An adaptive non linear least-squares algorithm. ACM Trans. Math. Software, 7(3): 348-368.

Dennis, J. E. Jr॰; Gay, D. M•; Welsch, R. E. (1981b). Algorithm 573: NL2SOL - an adaptive non linear least squares algorithm [E4]. ACM Trans. Math. Sof tware, $7(3)$ : 369-383.

Dennis, J. E. Jr.; Schnabel, R. B. (1983). Numerical methods for unconstrained optimization and nonlinear equations. New Jersey: Prentice-Ha11, In c.

Donaldson, J. R•; Tryon, P. V. (1983). Introduction to STARPAC, the St andards time series and regression package. Nat. Bur. St and. (U.S.) Tech. Note 1068-1.

Dongarra, J. J.; Moler, C. B.; Bunch, J. R.; Stewart, G. W. (1979). LINPACK users' guide. Philadelphia: SIAM.

Draper, N. R.; Smith, H. (1981). Applied regression analysis, second edition. New York: John Wiley and Sons.

Filliben, J. J. (1977). User's guide to the DATAPAC data analysis package. Un published.

Fox, P. A.; Ha11, A. D.; Schryer, N. L. (1978a). Algorithm 528: framework for a portable library [z]. ACM Trans. Math. Software, 4(2): 177-188.

Fox, P. A.; Hall, A. D.; Schryer, N. L. (1978b). The PORT mathematical subroutine library. ACM Trans. Math. Software, 4(2): 104-126.

Gi11, P. E•; Murray, W•; Wright, M.H. (1981). Practical optimization. New York: Academic Press. 
Hoaglin, D. C.; Welsch, R. E. (1978). The hat matrix in regression and ANOVA. American Statistician, 32: 17.

Hogben, D.; Peavy, S. T.; Varner, R. N. (1971). OMNITAB II user's reference manual. Nat. Bur. Stand. (U.S.) Tech. Note 552.

Kennedy, W. J. Jr.; Gentle, J. E. (1980). Statistical computing. New York: Marce1 Dekker, Inc.

Lawson, C.; Hanson, R.; Kincaid, D.; Krogh, F. (1979). Basic linear algebra subprograms for FORTRAN usage. ACM Trans. Math. Software, 5(3): 308-371.

Ryder, G. B. (1974). The PFORT verifier. Software Practice and Experience, $4(4)$; October-December: 359-377.

Schnabel, R. B. (1982). Finite difference derivatives - theory and practice. (Unpublished - available from J. R. Donaldson.)

Tryon, P. V.; Donaldson, J. R. (1978). STATLIB: A library of Fortran subroutines for statistical analysis of experimental data. (Unpublished - available from J. R. Donaldson.) 
NBS-I14A REV. $2-8 \mathrm{C}$

U.S. DEPT. OF COMM.

1. PUBLICATION OR REPORT NO.

BIBLIOGRAPHIC DATA

NPS TN-1068-2

2. Performing Organ. Report No. 3. Publication Date

October 1983

TITLE AND SUBTITLE

Nonlinear Least Squares Regression U'sing STARPAC, The Standards Time Series and Regression Package

5. $A \cup T H O R(S)$

Janet R. Donaldson and Peter V. Tryon

6. PERFORMING ORTANIZATION (If joint or other than NBS, see instructions)

7. Contract/Grant No.

NATIONAL BUREAU OF STANDARDS

DEPARTMENT OF COMMERCE

WASHINGTON, D.C. 20234

8. Type of Report \& Period Covered

Report 非 2 in a series

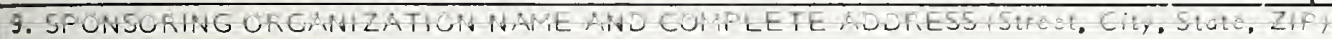

10. SUPPLEMENTARY NOTES

Document describes a computer program; SF-185, FIPS Software Summary, is attached.

11. ABSTRACT (A 200-word or less factual summary of most significant information. If document includes a significant

titliography or literature survey, mention it here)

STARPAC, the Standards Time Series and Regression Package, is a library of Fortran subroutines for statistiçal data analysis develope $\bar{s}$ hy the Statistical Engineering Division (SED) of the National Rureau of Standards (NBS), Roulder, Colorado. Earlier versions of this library were distributed by the SED under the name STATLIB ITryon and Donaldson, 1978]. STARPAC incorporates many changes to STATLIB, including additional statistical techniques, improved algorithms and enhanced portahility. STARPAC emphasizes the statistical interpretation of results, and, for this reason, comprehensive printed reports of auxiliary statistical information, often in graphical form, are automatically provided to augment the basic statistical computations performed by each user-callable STARPAC subroutine. STARPAC thus provides the hest features of many stand-alone statistical software programs within the flexible environment of a subroutine library. This Note documents 16 subroutines for nonlinear least squares regression. Twelve of these compute the least squares estimates, performing either weighted or unweighted analysis with either numerically approximated or user-supplied (analytic) derivatives. The other four are user-callable subroutines for two procedures used within the estimation code: the first selects optimum step sizes for approximating the partial derviatives of the model; and the second checks the validity of a user-supplied derivative subroutine.

12. KEY WORDS (Six to iwelve entries; alphabetical order; capitalize only proper names; and separate key words ty semicolons) derivative checking; NL2SOL; nonlinear least squares; nonlinear regression: quasi-Newton methods; STARPAC; statistical computing; statistical subroutine library; statistics; derivative step size selection; weighted nonlinear least squares.

13. AVAILABILITY

Unlimited

$\square$ For Official Distribution. Do Not Release to NTIS

X] Order From Superintendent of Documents, U.S, Government Printing Office, Washington, D.C. 20402.

$\square$ Order From National Technical Information Service (NTIS), Springfield, VA. 22I6I

14. NO. OF

PRINTED PAGES

USOCOTERENT PRINTING OFFICE: 1983-776876/62 





\section{NBS TECHNICAL PUBLICATIONS}

\section{PERIODICALS}

JOURNAL OF RESEARCH-The Journal of Research of the National Bureau of Standards reports NBS research and development in those disciplines of the physical and engineering sciences in which the Bureau is active. These include physics, chemistry, engineering, mathematics, and computer sciences. Papers cover a broad range of subjects, with major emphasis on measurement methodology and the basic technology underlying standardization. Also included from time to time are survey articles on topics closely related to the Bureau's technical and scientific programs. As a special-service to subscribers each issue contains complete citations to all recent Bureau publications in both NBS and nonNBS media. Issued six times a year. Annual subscription: domestic \$18; foreign \$22.50. Single copy, \$5.50 domestic; $\$ 6.90$ foreign.

\section{NONPERIODICALS}

Monographs-Major contributions to the technical literature on various subjects related to the Bureau's scientific and technical activities.

Handbooks-Recommended codes of engineering and industrial practice (including safety codes) developed in cooperation with interested industries, professional organizations, and regulatory bodies

Special Publications-Include proceedings of conferences sponsored by NBS, NBS annual reports, and other special publications appropriate to this grouping such as wall charts, pocket cards, and bibliographies.

Applied Mathematics Series-Mathematical tables, manuals, and studies of special interest to physicists. engineers, chemists, biologists, mathematicians, computer programmers, and others engaged in scientific and technical work.

National Standard Reference Data Series-Provides quantitative data on the physical and chemical properties of materials, compiled from the world's literature and critically evaluated. Developed under a worldwide program coordinated by NBS under the authority of the National Standard Data Act (Public Law 90-396)

NOTE: The principal publication outlet for the foregoing data is the Journal of Physical and Chemical Reference Data (JPCRD) published quarterly for NBS by the American Chemical Society (ACS) and the American Institute of Physics (AIP). Subscriptions, reprints, and supplements available from ACS, 1155 Sixteenth St., NW, Washington. DC 20056
Building Science Series-Disseminates technical irformation developed at the Bureau on building materials, components, systems, and whole structures. The series presents research results, test methods, and performance criteria related to the structural and environmental functions and the durability and safety characteristics of building elements and systems.

Technical Notes-Studies or reports which are complete in themselves but restrictive in their treatment of a subject. Analogous to monographs but not so comprehensive in scope or definitive in treatment of the subject area. Often serve as a vehicle for final reports of work performed at NBS under the sponsorship of other government agencies.

Voluntary Product Standards-Developed under procedures published by the Department of Commerce in Part 10, Title 15. of the Code of Federal Regulations. The standards establish nationally recognized requirements for products, and provide all concerned interests with a basis for common understanding of the characteristics of the products. NBS administers this program as a supplement to the activities of the private sector standardizing organizations.

Consumer Information Series-Practical information, based on NBS research and experience, covering areas of interest to the consumer. Easily understandable language and illustrations provide useful background knowledge for shopping in today's technological marketplace.

Order the above NBS publications from: Superintendent of Documents. Government Printing Office, Washington, DC 20402

Order the following NBS publications-FIPS and NBSIR's-from the National Technical Information Service. Springfield, VA 22161.

Federal Information Processing Standards Publications (FIPS PUB)-Publications in this series collectively constitute the Federal Information Processing Standards Register. The Register serves as the official source of information in the Federal Government regarding standards issued by NBS pursuant to the Federal Property and Administrative Services Act of 1949 as amended. Public Law 89-306 (79 Stat. 1127), and as implemented by Executive Order 11717 (38 FR 12315, dated May 11, 1973) and Part 6 of Title 15 CFR (Code of Federal Regulations).

NBS Interagency Reports (NBSIR) - A special series of interim or final reports on wiork performed by NBS for outside sponsors (both government and non-government). In general, initial distribution is handled by the sponsor: public distribution is by the National Technical Information Service, Springfield, VA 22161, in paper copy or microfiche form 


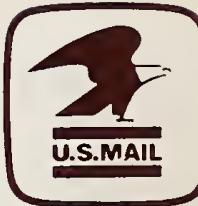

Washington, D.C. 20234

Official Business

Penalty for Private Use $\$ 300$

POSTAGE AND FEES PAID US DEPARTMENT OF COMMER COM-215 SPECIAL FOURTH-CLASS RATS BOOK 